3

\title{
Imaging for the evaluation of endometriosis and adenomyosis
}

\author{
Caterina Exacoustos ${ }^{\mathrm{a}, *}$, Lucia Manganaro ${ }^{\mathrm{b}}$, Errico Zupi ${ }^{\mathrm{c}}$

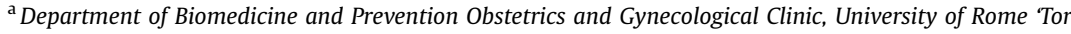 \\ Vergata', Italy \\ ${ }^{\mathrm{b}}$ Department of Radiological, Oncological and Pathological Sciences, University of Rome 'Sapienza' Italy \\ ${ }^{\mathrm{c}}$ Department of Molecular and Developmental Medicine, University of Siena, Italy
}

Keywords:

endometrioma

deep infiltrating endometriosis

pouch of Douglas obliteration

pelvic adhesions

adenomyosis

junctional zone
Endometriosis affects between 5 and 45\% of women in reproductive age, is associated with significant morbidity, and constitutes a major public health concern. The correct diagnosis is fundamental in defining the best treatment strategy for endometriosis. Therefore, non-invasive methods are required to obtain accurate diagnoses of the location and extent of endometriotic lesions. Transvaginal sonography and magnetic resonance imaging are used most frequently to identify and characterise lesions in endometriosis. Subjective impression by an experienced sonologist for identifying endometriomas by ultrasound showed a high accuracy. Adhesions can be evaluated by real-time dynamic transvaginal sonography, using the sliding sign technique, to determine whether the uterus and ovaries glide freely over the posterior and anterior organs and tissues. Diagnosis is difficult when ovarian endometriomas are absent and endometriosis causes adhesions and deep infiltrating nodules in the pelvic organs. Magnetic resonance imaging seems to be useful in diagnosing all locations of endometriosis, and its diagnostic accuracy is similar to those obtained using ultrasound. Transvaginal ultrasound has been proposed as first line-line imaging technique because it is well accepted and widely available. The main limitation of ultrasound concerns lesions located above the rectosigmoid junction owing to the limited field-of-view of the transvaginal approach and low accuracy in detecting upper bowel

\footnotetext{
* Corresponding author. Universitá degli Studi di Roma 'Tor Vergata', Department of Biomedicine and Prevention, Obstetrics and Gynecological Clinic, Ospedale Generale S. Giovanni Calibita , Fatebenefratelli, Isola Tiberina 1, 00186 Roma, Italy. Tel.: +39 $066837416,+393358303967$.

E-mail address: caterinaexacoustos@tiscali.it.
} 
lesions by transabdominal ultrasound. A detailed non-invasive diagnosis of the extension in the pelvis of endometriosis can facilitate the choice of a safe and adequate surgical or medical treatment.

() 2014 Elsevier Ltd. All rights reserved.

\section{Introduction}

Endometriosis is estimated to affect between $5 \%$ and $45 \%$ of women of reproductive age, is associated with significant morbidity, and constitutes a major public health concern [1,2]. Symptoms of women with pelvic endometriosis are chronic pelvic pain, dysmenorrhoea, dyspareunia, dyschezia, urinary symptoms, and infertility. [3]

Three different forms of endometriosis exist: ovarian endometriosis (endometrioma), peritoneal endometriosis and adhesions, and deep endometriosis. Pelvic endometriosis, especially in severe stages, is strongly associated with adenomyosis, which plays an important role in causing dysmenorrhoea, menorrhagia, and infertility in women with endometriosis.

Ovarian lesions are the most frequent localisation of endometriotic tissue, causing typical ovarian cysts. Deep infiltrating endometriosis (DIE) is defined as an endometriotic lesion infiltrating the peritoneum and penetrating into the retroperitoneal space or the wall of the pelvic organs to a depth of at least $5 \mathrm{~mm}$ [4], and affects between 4 and 37\% of women with endometriosis. These different forms of presentation are likely to have different imaging patterns, which may cause specific imaging diagnostic problems. Several systems scores have been used to stage the extension of endometriosis in relation to different locations inside the pelvis. The most common system used to evaluate the disease is the revised classification system of the American Society of Reproductive Medicine (rASRM), which followed the American Fertility Society (AFS) score [5]. As with other systems, this classification does not consider adenomyosis as part of the disease, which remains after surgical treatment of extrauterine lesions, with persistence of symptoms related to pelvic endometriosis.

The interval between the onset of first symptoms and clinical diagnosis of endometriosis is about 7-10 years [6]. The main diagnostic problems for endometriosis are the detection of the disease, especially in the absence of an endometriotic cyst or in the case of minimal lesions, and also the evaluation of the extent of the disease. The patient's history and symptoms, a pelvic examination, along with the experience of the sonographer or radiologist, could improve diagnostic accuracy in the diagnosis of pelvic endometriosis.

The correct diagnosis is fundamental to defining the best treatment strategy for endometriosis; therefore, non-invasive methods are required to obtain accurate diagnoses of the location and extent of endometriotic lesions. Two imaging modalities are used most frequently to identify and characterise lesions in endometriosis: transvaginal sonography and magnetic resonance imaging.

Transvaginal ultrasonography has been proposed as the first line-line imaging technique because it allows extensive exploration of the pelvis; it is well accepted and widely available.

Magnetic resonance imaging (MRI) is used as a second-line of investigation in the study of the female pelvis. The role of MRI in the evaluation of endometriosis, especially DIE, has been widely demonstrated. MRI is carried out in selected women according to the outcome of transvaginal ultrasound imaging and the severity of symptoms. Many investigators have studied the role of MRI in the evaluation of deep implants located in the anterior compartment, recto-vaginal septum, posterior vaginal fornix, and bowel wall, especially for the lesions located above the rectosigmoid junction.

Other diagnostic procedures, such as rectal sonography, barium enema, or computed tomography urography play complementary roles in the identification of endometriosis, depending on the site affected, and could be useful in the choice of surgical approach. Transabdominal ultrasound is not accurate in detecting endometriosis, mainly because of bowel gas and adhesions that may reduce the ability to evaluate the pelvic organs. In particular, DIE mostly has retroperitoneal or bowel lesions, which are difficult to see with transabdominal ultrasound probes [7].

A detailed non-invasive examination of the pelvis to assess the extension of the endometriosic lesions can facilitate the choice of a safe and adequate surgical or medical strategy [4]. 


\section{Endometrioma}

\section{Diagnostic imaging and implications for treatment}

The correct diagnosis of an endometrioma is important because an association exists between endometrioma, endometriosis, and chronic pelvic pain and subfertility. Moreover, an endometrioid adenocarcinoma or clear-cell carcinoma may develop in endometriomas [8-10].

Transvaginal sonography has dramatically improved the ability to diagnose ovarian lesions, providing reliable criteria and indications for surgery [11,12]. The bizarre echogenicity of some endometriomas can erroneously suggest malignancy and vice versa. Therefore, diagnostic criteria to distinguish ovarian endometriomas from other ovarian lesions seem to be of utmost importance. Endometriomas were treated in the past mostly by surgical excision. In cases of recurrence, surgery was repeated, subsequently causing great problems on ovarian reserve. For ovarian endometriosis, first-line treatment is medical treatment and surgery carried out only in cases of suspected malignancy or persistence of symptoms and infertility [13].

\section{Ultrasound features}

\section{Endometrioma}

Ultrasound characteristics of endometriomas have been described in several studies, and an attempt has been made to define their typical ultrasound features [14-16]. The 'typical' endometrioma is a unilocular or multilocular (less than five locules) cyst, with homogeneous low-level echogenicity (ground glass echogenicity) of the cyst fluid. Because endometriomas are usually poorly vascularised, power or color Doppler ultrasound examination might help to achieve a correct diagnosis [17]. (Fig. 1). Morphologic features other than the 'typical' ones have also been described [17]. Guerriero et al. [17] and Dogan et al. [18] were the first to conduct studies to characterise these atypical endometriomas. The algorithm by Guerriero et al. [17] defines an endometrioma as either a unilocular cyst with ground glass echogenicity and no to moderate vascularisation ('typical' endometrioma) (Fig. 1) or as a unilocular cyst with ground glass echogenicity and papillary projections (protrusion of solid tissue into the cyst lumen with a height of $3 \mathrm{~mm}$ or more), and no flow inside the papillary projection ('atypical' endometrioma). In fact, these are not true papillations of solid tissue but images created from blood clots or fibrin lying adjacent to the cyst wall, showing a more regular surface and round shape of the protrusion (Fig. 2).

According to the algorithm by Guerriero et al. [17], a unilocular cyst with ground glass echogenicity and strong vascularisation or a unilocular-solid cyst with ground glass echogenicity and a papillary projection with detectable flow or strong vascularisation are classified as non-endometriomas.

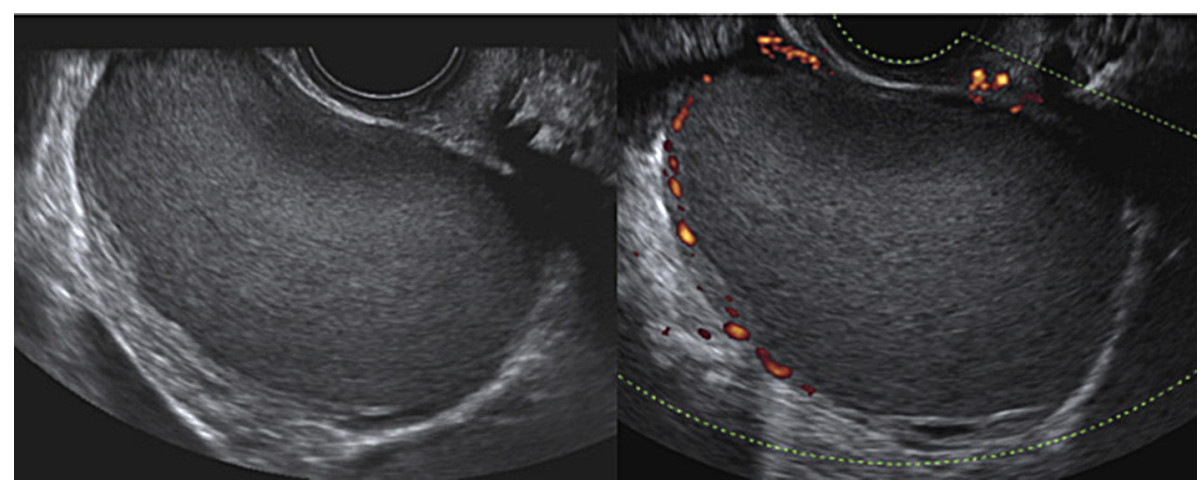

Fig. 1. Typical ultrasound appearance of an ovarian endometrioma: a unilocular cyst with ground glass echogenicity and little to moderate vascularisation. Note the normal ovarian tissue around the cyst. 


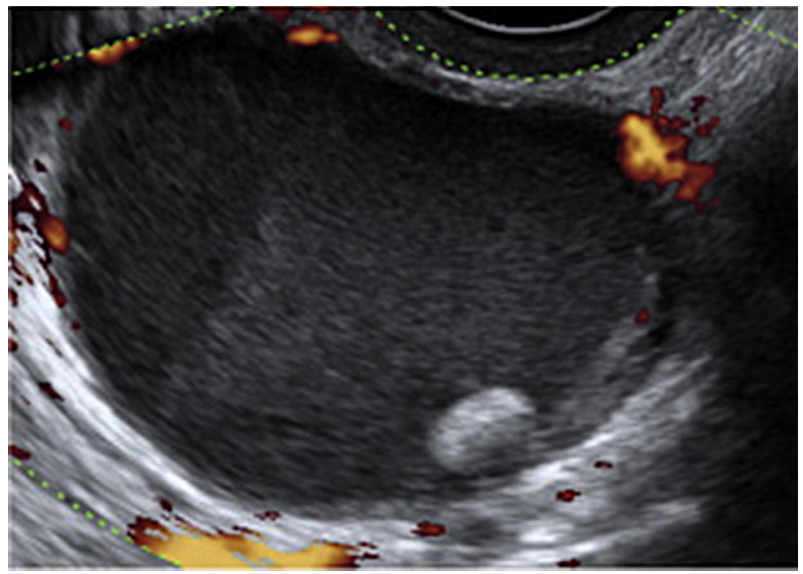

Fig. 2. Atypical ultrasound appearance of an ovarian endometrioma: a unilocular cyst with ground glass echogenicity, internal papillation and no vascularisation in the papillary projection. This is not a true papillations but hyperechoic tissue consisting of blood clots or fibrin lying adjacent to the cyst wall.

Almost $50 \%$ of the endometriomas had other ultrasound characteristics than the typical 'unilocular cyst with ground glass echogenicity of the cyst fluid'.

The rule: 'premenopausal status, ground glass echogenicity, one to four locules and no papillations with detectable blood flow' characterise endometriomas reasonably well, but not as well as subjective impression [19]. Serum CA-125 levels are not generally useful in distinguishing endometriomas from other benign tumours and malignancies [19]. They could possibly help to distinguish endometriomas from other benign lesions [13].

In addition, ultrasound appearance of endometriomas differ between pre- and post-menopausal women. Endometriomas in the postmenopausal women are less frequently unilocular cysts, and are less likely to exhibit ground glass echogenicity [19]. Instead, they are more often multilocularsolid tumours, and more frequently exhibit anechoic cyst fluid or cyst fluid with mixed echogenicity.

Endometriomas may be misinterpreted, potentially because of the complex echotexture, thick walls, and solid echogenic appearance of haemorrhagic clots within the endometrioid cystic cavity, which mimic different dermoid cyst patterns or malignancy. Also, some hyperechoic solid masses (e.g. fibroids and fibrothecomas) can be misdiagnosed as endometriotic cysts. Inversely, some endometriomas can be mistaken for serous cysts, dermoids, and suspected ovarian malignant tumours. The use of colour or power Doppler detecting the presence, number, and distribution of vessels in the solid echogenic protrusions of the cyst wall seems to be useful in differentiating endometriomas from malignant lesions $[10,16,17]$. Unfortunately the use of colour Doppler evaluation has some limitations , because it requires optimal colour Doppler settings, a high quality of the colour Doppler function of the ultrasound equipment used and an experienced ultrasound examiner. Therefore, the absence of vascularisation does not guarantee that the cyst is benign.

The clinical rule that considers endometrioma a cyst with ground glass echogenicity 1-4 septa, with no solid parts, in a premenopausal woman, is useful in most clinical settings because it allows the examiner to skip the colour Doppler assessment of the mass. This clinical rule has almost as good discriminatory power as the statistically optimal rule, but its sensitivity is lower [19]. Finally all the principal rules have a sensitivity ranging from $62-73 \%$, a specificity of $94-98 \%$, and a positive predictive value of 76-89\% [19]. Subjective impression by an experienced sonologist was better for identifying endometriomas (positive predictive value $88.5 \%$, positive likelihood ratio 30.2 , sensitivity $81 \%$, specificity $97 \%$ ) $[10,19]$. This is probably because the ultrasound examiner uses other available clinical information (e.g. pain and dysmenorrhoea) when suggesting a diagnosis, taking into account other ultrasound findings. 


\section{Endometriomas and malignancy}

Subjective impression can lead to the misclassification of malignancies as endometriomas in 0.2$0.9 \%$ of cases $[10,11,19]$. Ultrasound characteristics of endometriomas differ in pre- and postmenopausal women. Masses in postmenopausal women, whose cystic contents have a ground glass appearance, have a high risk of malignancy.

Endometriomas could serve as precursors of endometrioid borderline ovarian tumours. Endometrioid borderline ovarian tumours have the potential to progress to low-grade invasive carcinoma. Although clear-cell borderline ovarian tumours have been associated with endometriosis, a stepwise molecular pathway for the progression of endometriosis to clear carcinoma has not yet been identified [20].

Borderline tumours and carcinomas arising from endometrioid cysts show a vascularised solid component at ultrasound examination (Fig. 3). The presence of typical sonographic features for ovarian malignant lesions suggests that benign endometrioid cysts and malignant and borderline tumours arising from endometriosis might be easier to assess by an expert ultrasound examiner compared with normal ovarian masses [9]. This is not the case with ovarian cysts found during pregnancy, were differentiation between borderline tumours and decidualised endometriotic cysts can be more difficult. Ultrasound examiners should always take into account the phenomenon of decidualisation, which is a major contributory factor to incorrect diagnosis in pregnant women [21]. Most decidualised endometriomas $(82 \%)$ were described as manifesting vascularised rounded papillary projections with a smooth contour in an ovarian cyst with one or more cyst locules and ground glass, or low level echogenicity of the cyst fluid [22].

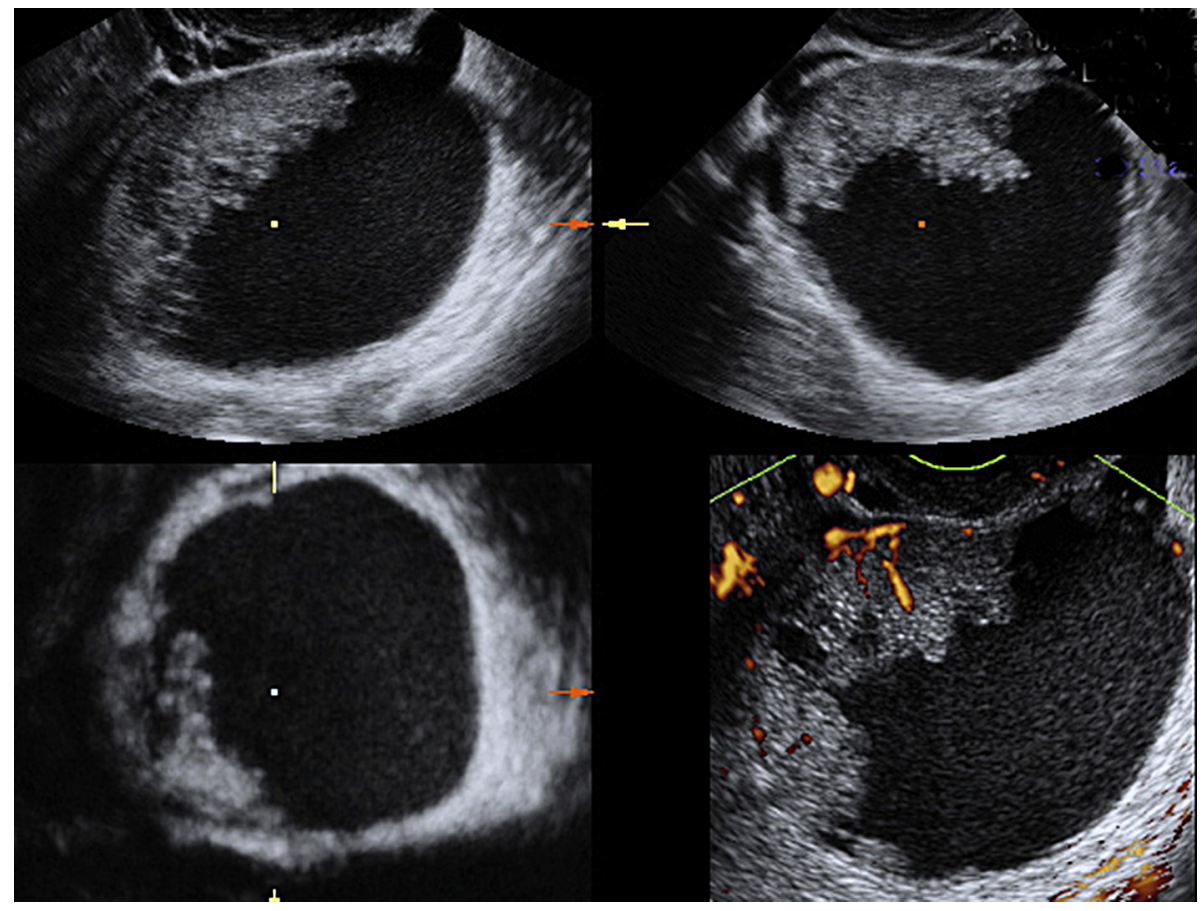

Fig. 3. Three-dimensional ultrasound and power-Doppler image of an endometrioid borderline tumour. Note the irregular papillations and the vascularisation in the papillary projections. These are true papillations composed of borderline malignant solid tissue. 


\section{Endometriomas and tubal pathology}

Where endometrioma or pelvic endometriosis are present, the salpinges can also often be affected by the disease. Adhesions can alter the normal tubal course or occlude the tube, and endometriotic foci can affect the tubal walls. Consequently, a sactosalpinx can be observed near the endometrioma. The typical aspect is a dilated Fallopian tube with thick walls and incomplete septa, seen as a cogwheelshaped structure ('cogwheel' sign) and visible in the cross-section, with a fluid-dense content [23]. Sometimes, the chronic disease can cause a hydrosalpinx with the typical 'beads-on-a-string' sign, defined as hyperechoic mural nodules measuring about $2-3 \mathrm{~mm}$ and seen on cross-section of the fluidfilled distended structure [23]. (Fig. 4). The presence of hydrosalpinx in infertile women is an indication for surgical removal of the Fallopian tubes [24]. It is important to assess tubal status in cases of endometrioma or DIE by ultrasound.

Endometriosis that affects the ovary and Fallopian tubes can create a tubo-ovarian complex, in which the ovaries and tubes are identified and recognised, but the ovaries cannot be separated by pushing the tube with the vaginal probe. Rarely, the normal architecture of one or both the adnexa and tubes cannot be recognised, and a conglomerate of endometric cysts form; however, neither the ovary nor the tubes can be separately identified [23].

\section{Magnetic resonance imaging features}

\section{Endometrioma}

Magnetic resonance imaging for endometriotic ovarian cyst is requested in selected cases if ultrasound outcome is inconclusive, if malignant transformation is suspected, or both. It presents a high specificity in diagnosing endometriomas (98\%) [25], owing to its ability to characterise haemorrhage. 'Shading' is a specific sign of endometrioma; it is caused by old blood products, which contain extremely high iron and protein concentrations. These haemorrhagic cysts typically show high signal intensity on T1-weighted images and variable low signal intensity on T2-weighted images. Shading varies from a faint signal to a no signal at all. Solid components, clots, thick septa, and fluid-fluid levels

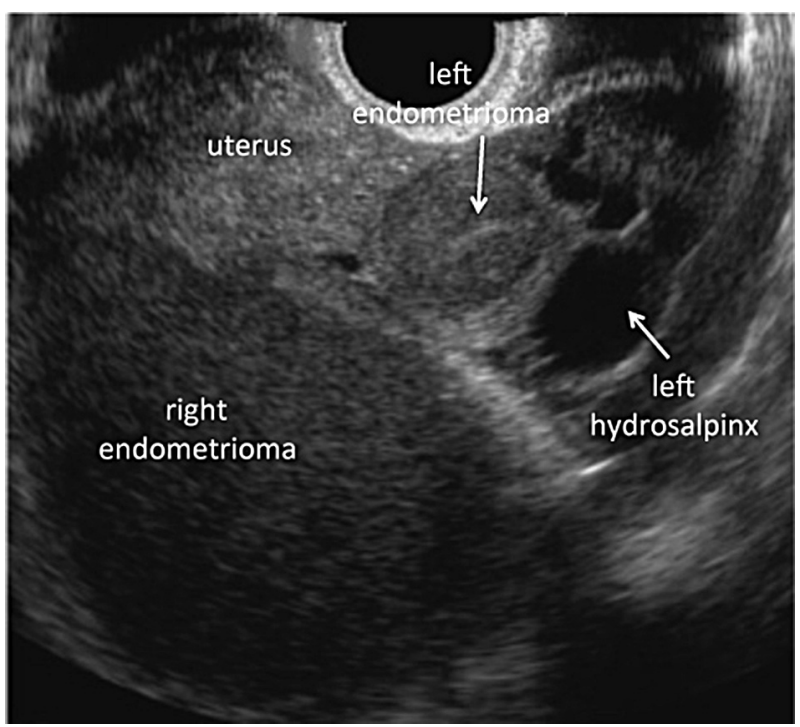

Fig. 4. Ultrasound image from a woman with hydrosalpinx attached to the left ovary with a small endometrioma and the right ovary with a larger endometrioma adherent posteriorly to the uterus and to the left tubo-ovarian complex. 
may also be observed at MRI [26]. In these cases, the investigation should be completed with contrastenhanced sequences to assess the risk of neoplastic transformation.

\section{Tubal endometriosis}

Hyperintense tubal fluid seen on T1-weighted images, with or without evidence of endometriosis elsewhere in the pelvis, is suggestive of hydrosalpinx associated with endometriosis. On T2-weighted MRI scans, signal intensity within the fallopian tube is generally high owing to the typical signal intensity of an endometrial cyst, which displays moderate to marked shading. Haematosalpinx has been reported to be an indicator of pelvic endometriosis, and may be the only imaging finding indicative of endometriosis [27].

\section{Adhesions}

\section{Diagnostic imaging and implications for treatment}

Ovarian endometriomas are frequently associated with other endometriotic lesions [28], such as adhesions and DIE, which are not easy to diagnose. Underestimation of extensive adhesions in women with endometriomas before surgery is one of the main reasons why surgery is often incomplete [28], leading to repeat operations.

Advances in endoscopic surgery have permitted laparoscopic treatment of ovarian cysts that previously required laparotomy [29] and, at present, laparoscopic management of ovarian endometriosis is the technique of choice. Adhesions and DIE lesions, however, remain the most important limitation in the surgical treatment of endometriosis [29]. This limitation obviously differs according to the surgeon's experience and skill. It seems important, therefore, that preoperative patient selection should be managed according to pelvic extension of the disease. Women with severe disease and extensive adhesions could, therefore, be referred to centres of excellence to ensure complete surgical excision, which requires particular laparoscopic surgical skills and experience [30].

\section{Ultrasound evaluation}

Endometriosis is often associated with the presence of pelvic adhesions. Ultrasound diagnosis in the evaluation of pelvic adhesions in the presence of ovarian endometriosis is a diagnostic challenge. This diagnostic impasse is particularly evident when ovarian endometriomas are absent and endometriosis causes adhesions and small nodules in the pelvic organs, which are difficult, if not impossible, to detect by ultrasound. It has been reported that peritoneal disease and adhesions are more common than ovarian disease [31]. Therefore, in women who are infertile or have chronic pelvic pain, and in the absence of ovarian endometriomas, it is important to look for sonographic signs of adhesions. Only a few studies have attempted to assess the ability of transvaginal sonographic examination to detect the presence of pelvic adhesions in women with pelvic endometriosis, and to assess their severity [32-35].

Normally, the uterus and ovaries are mobile and do not adhere to the surrounding tissues by palpation with the probe, abdominal palpation with the hand, or both. Movement of these organs can be seen on ultrasound (sliding sign). Usually, palpation with the probe, or abdominal palpation with the hand, can cause the ovaries or the uterus to adhere to adjacent structures (e.g. broad ligament, pouch of Douglas (POD), bladder, rectum, and parietal peritoneum). In this case, adhesions can be suspected. Sometimes, in the presence of pelvic fluid, fine septa (adhesions) can be seen between the ovary, the endometrioma, the uterus, or the peritoneum of the POD $[33,35]$. Endometrioma are usually fixed posteriorly to the uterus, in the POD. The cause of this, especially in cases of bilateral endometrioma, is that both ovaries are fixed posteriorly to the uterus and have adhered to the controlateral ovary (kissing ovaries) (Fig. 5)

To assess stages of the disease in the pelvis, the revised American Fertility Society classification for endometriosis [5] includes the presence and extension of adhesions, endometrioma size, and POD obliteration. Deep endometriotic lesions are not included in this classification. 


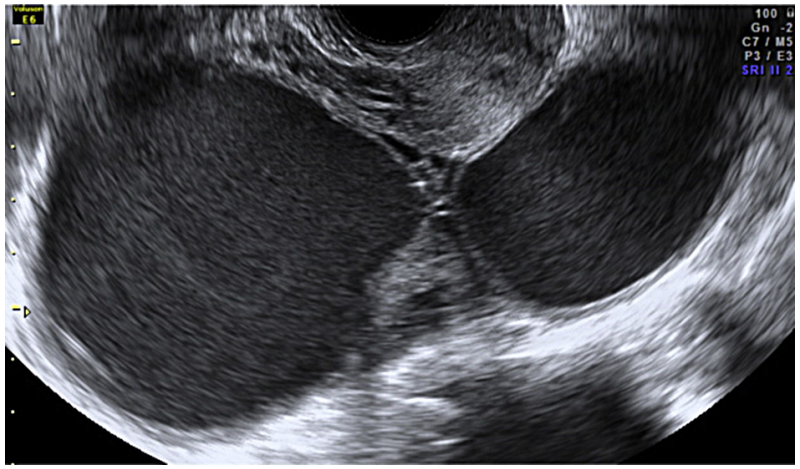

Fig. 5. Ultrasound image of bilateral endometriomas with both ovaries adherent to each other (kissing ovaries) and posteriorly to the uterus.

Recently, preoperative diagnosis of partial or complete obliteration of the POD has been described [36-39]. The POD is examined using real-time ultrasound imaging to determine the presence or absence of POD obliteration using the sliding sign. To assess the sliding sign, gentle pressure is placed against the cervix with the transvaginal probe to establish whether the anterior rectum glides freely across the posterior aspect of the cervix (posterior cervical region) and posterior vaginal wall.

A number of studies have been conducted on the accuracy of transvaginal sonography for the diagnosis of ovarian adhesions. Classification of the severity of ovarian adhesions as either minimal, moderate or severe, in accordance with the rAFS classification, is rare [5]. Okaro et al. [33] found that preoperative transvaginal sonographic 'soft markers' (i.e. site-specific tenderness and reduced ovarian mobility) and 'hard markers' (i.e. endometrioma, hydrosalpinx) in women with a history of chronic pelvic pain correlated with the presence or absence of endometriosis and adhesions at laparoscopy. Preoperative transvaginal and transrectal ultrasound have also been used to predict Stage 3 and 4 endometriosis (including pelvic adhesions) at laparoscopy, with a sensitivity and specificity of $86 \%$ and 82\%, respectively, for Stage 3 and 76\% and 91\%, respectively, for Stage 4 disease [35]. Guerriero et al. [32] used a technique of applying pressure between the uterus and ovary, and found that a combination of three features are suggestive of ovarian adhesions: blurring of the ovarian margin; the inability to mobilise the ovary on palpation (fixation); and an increased distance from the probe. Sensitivity and specificity of $89 \%$ and $90 \%$, respectively, were reported for fixation of the ovaries to the uterus.

The real-time preoperative dynamic transvaginal ultrasound examination of POD obliteration, using the sliding sign technique, seems to be useful in identifying women at increased risk for bowel endometriosis. Hudelist et al. [38] reported that a negative sliding sign on transvaginal sonography predicted DIE of rectum, with a sensitivity of $85 \%$, specificity of $96 \%$, and an accuracy of $93.1 \%$. Reid et al. [39] reported a sensitivity and specificity of $83.3 \%$ and $97.1 \%$, respectively, for prediction of POD obliteration.

\section{Magnetic resonance imaging evaluation}

Adhesions can be problematic in the evaluation of endometriosis. At MRI, adhesions may sometimes be identified as spiculated low-signal-intensity stranding on T1- and T2- weighted images with a different thickness. Often, only indirect signs of adhesions are present. These include angulation of bowel loop, skip of bowel diameter, elevation of the posterior vaginal fornix, posterior displacement of the uterus, ovaries, or both, loss of fat planes between the structures, hidrosalpinx, and loculated fluid collection. Kataoka et al. [40] reported a mean sensitivity of $77.8 \%$, a mean specificity of $50.0 \%$, and a mean accuracy of $76.3 \%$ for the detection of adhesions.

The use of MRI in the evaluation of endometriosis may, therefore, be difficult, and will require experience and specific training in pelvic imaging. The distortion of the structures and the hyperperistaltism of the bowel can cause diagnostic problems in visualising adhesions during MRI. 


\section{Deep infiltrating endometriosis}

\section{Diagnostic imaging and implications for the treatment of endometriosis}

Deep infiltrating endometriosis is the most severe form of endometriosis. It is associated with infertility or pain symptoms, including chronic pelvic pain, dysmenorrhoea, dyspareunia, dysuria, and dyschezia [4]. A wide spectrum of symptom severity exists, and the stage of endometriosis at laparoscopy is poorly correlated with the extent and severity of pain, often resulting in misdiagnosis or delay in diagnosis [3]. This is the case with DIE, as symptoms are not specific [2,3]. Bimanual pelvicgynaecologic examination may suggest the presence of DIE by the identification of tender nodules and fibrosis in the cul de sac, but it has poor accuracy in determining the extent of disease [3,36]. The current gold standard for a definitive diagnosis of endometriosis remains surgical evaluation with histological confirmation, even if deep retroperitoneal localisations have been missed on laparoscopy.

Laparoscopy, in the case of advanced extension of DIE, is a difficult procedure, with a high rate of complications. Therefore, medical treatment is the first choice, and surgery is only recommended in the presence of severe symptoms.

Knowledge before surgery of the anatomical position, size, number of DIE nodules, depth of infiltration of the nodules, and degree of stenosis of the bowel lumen, can help in planning the surgical procedure, in the provision of appropriate counselling to women who undergo the procedure, and in the choice of the right surgical team. For the surgical treatment of DIE, this will include a gynaecologic endoscopic surgeon with particular skills in DIE resection, a urologist and colorectal surgeon depending on the lesions site, or both. In the case of posterior DIE, when the recto-sigma is infiltrated by endometriotic tissue, the bowel is so retracted that even the upper segments can adhere to the posterior wall of the uterus, with complete disruption of normal anatomy. It is, therefore, difficult to distinguish between the rectum and sigma. From a surgical point of view, it is important that diagnostic imaging determinates the lowest limit of the nodule on the bowel wall, as the lower rectal lesions are more difficult to remove surgically by shaving or segmental resection, and have a higher complication rate.

Infiltration of the mucosal layer does not determine whether or not segmentary resection should be carried out; this decision more likely depends on the diameters of infiltrating tissue and the lumen stenosis it causes [4].

A systematic evaluation of the urinary tract in women with endometriosis is recommended because the prevalence of endometriotic lesions in the urinary tract may be underestimated [41]. Endometriosis of the ureter usually stems from endometriosis of the pelvic foci and ovarian endometriosis [41,42]. Extrinsic and intrinsic endometriosis are involved in the ureters: extrinsic represents $75-80 \%$ of cases, and is defined as the presence of endometrial tissue in the outer adventitia of the ureter. This occurs as a nodule encasing the ureter; intrinsic endometriosis represents $20-25 \%$ of cases, and is defined as the presence of endometrial tissue in the mucosal, muscular layer of the ureter, or both. Diagnostic imaging may show direct signs (e.g. a nodule or mass in the ureter along its course, and dilatation of the pelvic ureteral tract), or an indirect (e.g. ureteropelvic hydronephrosis superior to the suspected lesion). In the case of extrinsic compression, ureterolysis can be carried out, whereas a ureteral segmental resection and re-anastomosis is indicated in cases of intrinsic localisation of the disease. Therefore, an accurate evaluation of the urinary tract with computed tomography or MRI urography is mandatory if suspected ureteral involvement is found on transvaginal sonography.

Endometriosis is a chronic disease, and medical treatment, often combined with surgery, is the treatment of choice. Fedele et al. [43] showed that, in women who had previously undergone conservative surgery for endometriosis without excision of deep lesions further treated with levonorgestrel intrauterine device (LNG-IUD), dysmenorrhoea, which had been moderate or severe in all cases, during treatment were absent. Other medical options, such as vaginal danazol ( $200 \mathrm{mg} /$ day) were also tested on dysmenorrhoea and dyspareunia after surgery for DIE, showing a consistent and significant improvement in painful symptoms [44]. In a recent study of estroprogestin therapy, Mabrouk et al. [45] reported that combined oral contraceptive therapy may have a role to play in restraining the progression of dysmenorrhoea and dyspareunia, and the growth of deep endometriotic nodules. It has also 
been shown recently that medical treatment performed significantly better in relieving pain in women without DIE compared with women with recto-vaginal lesions [46].

It seems, therefore, that as much detail as possible about the spread and localisation of the disease are needed by the surgeon and clinician responsible for medical treatment. The careful evaluation of clinical and diagnostic imaging findings gives clinicians the opportunity to decide the best surgical approach, the possible need to involve surgical specialists other than a gynaecologic surgeon (e.g. colorectal surgeon or urologist), so that management of the disease can be tailored correctly, and patients can be informed of the extent of their disease and the therapeutic options available.

\section{Ultrasound evaluation of deep infiltrating endometriosis}

Transvaginal sonography can evaluate all potential locations of DIE in the anterior (bladder) or posterior-lateral compartment. These include the rectovaginal septum, uterosacral ligaments, torus uterinum (i.e. tissue behind the cervix in the mid-sagittal plane between the uterosacral ligaments), posterior vaginal fornix, rectum and rectosigmoid junction, and parametria and ureteral involvement.

Endometriotic nodules of the bladder and the rectum can be evaluated with a transvaginal probe and, if necessary, a transrectal examination with the same convex probe can be carried out. During the transrectal examination, a fluid contrast medium can be inserted into the vagina (Fig. 6) to better visualise the recto-vaginal septum.

Transabdominal ultrasound does not accurately detect DIE, mainly because bowel gas reduces the ability to evaluate abdominal retroperitoneal or small bowel lesions, which are difficult to detect with transabdominal ultrasound probes. Only endometriotic nodules of the abdominal wall can be easily evaluated by a high-frequency transabdominal probe.

Deep nodes appear as hypoechoic lesions, linear or nodular retroperitoneal thickening with irregular borders, and few vessels at power Doppler evaluation [47-50].

Women with suspected endometriomas associated with deep endometriosis, in particular those with a frozen pelvis or recto-vaginal or bladder nodules, should fist undergo a detailed examination of the pelvis to evaluate the anatomy of the uterus and the adnexa, both in the sagittal and horizontal plane, with gentle probe movements to assess the presence of adhesion between them. Transvaginal sonographic examination is based on a detailed evaluation of organ and tissues dividing the pelvis in the anterior and posterior compartment according to the DIE classification by Chapron et al. [51].

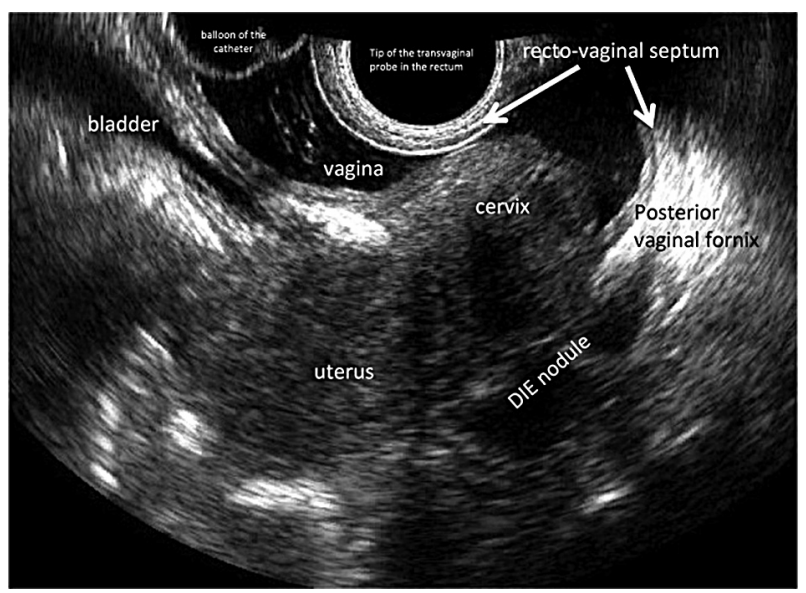

Fig. 6. Sonovaginography: visualisation of the vagina with transvaginal probe positioned in the rectum. The vagina is filled with saline solution through a Foley catheter with its balloon placed in the lower part of the vagina; note the wall of the vaginal posterior fornix, the cervix in the vagina, the recto-vaginal septum, and the retrocervical nodule of deep infiltrating endometriosis not invading the vagina but infiltrating the lower part of the rectal wall and the left uterosacral sacral ligament. 


\section{Deep infiltrating endometriosis of anterior compartment (bladder)}

Patients are asked not to empty their bladder completely before a transvaginal scan. The slightly filled bladder improves visualisation of the structure of the walls and presence of endometriotic nodules. Nodules appear as round shaped lesions, with or without cystic areas, with regular and irregular margins of the bladder wall, bulging towards the lumen most frequently on the posterior bladder wall close to the vesico-uterine pouch (Fig. 7). Bladder adhesions of the vesico-uterine pouch are evaluated by the presence or absence of the sliding sign between the uterus and the bladder. Bladder endometriosis is considered only in cases of infiltration of the bladder wall and not in cases of adhesions or superficial peritoneal implants on the bladder serosa.

\section{Deep infiltrating endometriosis of the posterior-lateral compartment}

Transvaginal and transrectal sonography (if needed) can be used to assess the vagina accurately, particularly the areas of the posterior and lateral vaginal fornixes, the retro-cervical area with torus uterinum, uterosacral sacral ligaments, the parametria laterally, and the recto-vaginal septum. In the case of endometriotic lesions of uterosacral ligaments and homolateral parametria, special attention needs to be paid to ureteral evaluation in the paracervical tract. To assess rectal wall infiltration, if suspected, transrectal evaluation with the transvaginal probe could be carried out. Special attention has to be paid to the pain felt by the women so that a careful evaluation of all the painful sites can be carried out by gently pressing the probe ('tenderness-guided' ultrasonography) [52]. Ultrasound findings are presented below.
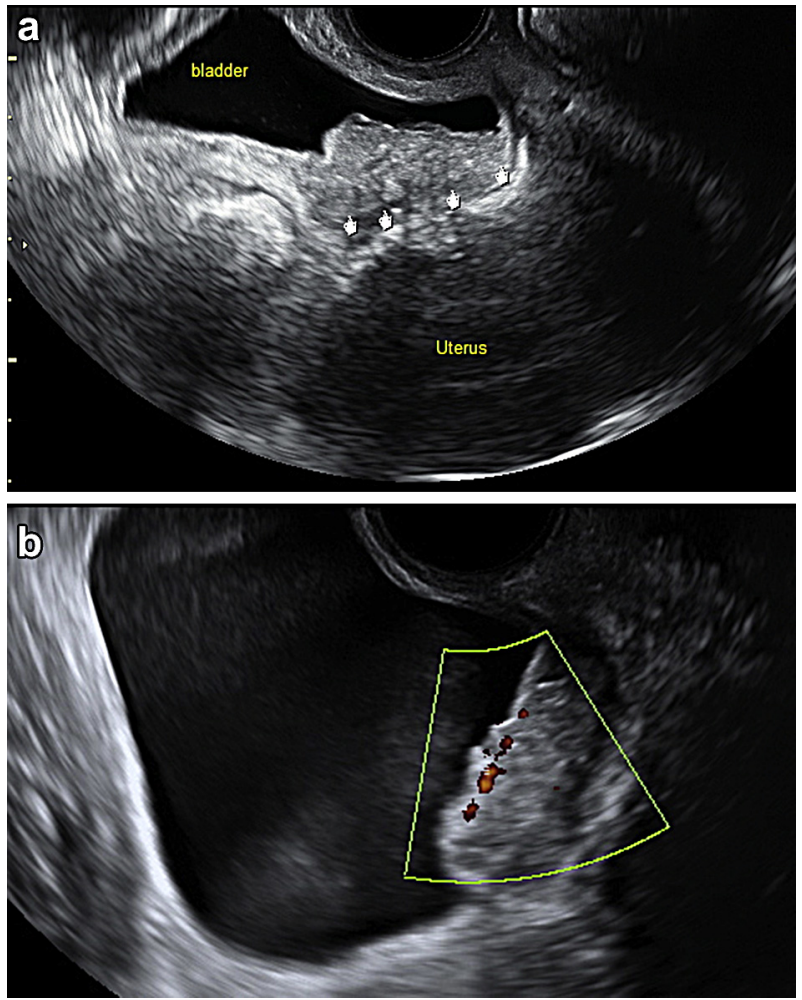

Fig. 7. Ultrasound image of an endometriotic bladder nodule. The slightly filled bladder makes it possible to see the irregular margins of the hyperechoic lesion bulging into the lumen of the bladder, infiltrating the posterior bladder wall and the vesicouterine pouch (hands). The nodule is attached to the uterine anterior wall (a). The same bladder lesion evaluated by power Doppler showing only few vessels inside the lesion (b). 
Vagina. In the vagina, nodular thickening of the posterior vaginal fornix takes place, which does not become thinner with probe compression. The insertion of saline solution into the vagina (sonovaginography) can improve visualisation of these lesions [53]. (Fig. 6) An increase in the amount of ultrasonographic gel inside the probe's cover can also improve visualisation of the vaginal walls and posterior and anterior fornix [52]. The low reported accuracy of transvaginal sonography in detecting vaginal endometriosis [36,47] confirms that digital gynaecological examination could be a better alternative to transvaginal sonography. In the recto-vaginal septum, nodules replacing the normal hyperechoic aspect of the tissue between the vagina and the rectum are present below the horizontal plane passing through the lower border of the posterior lip of the cervix [47].

In the torus, a nodular image with irregular margins behind the cervix is found in the mid-sagittal plane. The endometriotic lesion in uterosacral ligaments is visible near the insertion on the posterior lateral cervix wall, as a nodule with regular or stellate margins or as hypoechoic linear thickening.

In cases of endometriotic lesions involving the uterosacral ligament, special attention must be paid to the parametrium and to the pelvic ureteral evaluation, particularly in the paracervical area. Parametria are examined lateral to the uterine cervix firstly on the sagittal planes moving the probe from the lateral sites where the parametrium is attached to the cervix, to the uterine vessels bifurcation, to the lateral pelvic wall, and then on the transverse planes moving the probe from the uterine isthmus to the external cervical orifice. Parametrial involvement is seen as an infiltrating hypoechogenic irregular tissue, and can be medially delimited from the cervical vascular plexuses using colour or power Doppler.

Ureter. Pelvic ureteral dilatation can be easily seen by transvaginal sonography as a tubular anechoic image (Fig. 8) with or without movements in the parametrial tissue, similar to a blood vessel but with negative Colour or power Doppler signs. In the case of extrinsic compression without stenosis of the ureter, the transvaginal sonographic diagnosis is more difficult. The distal part of the ureter can be identified adjacent to the bladder trigon, and followed laterally to the cervix, to the pelvic brim and to the level where it crosses the common iliac vessels [54]. An extrinsic compression also without ureteral dilatation could be suspected in cases in which a DIE lesion is located close to the ureter. The hypothesis of ureteral involvement suggests a specific and accurate evaluation at the time of surgery, and, in these cases, transabdominal ultrasound to evaluate the renal pelvis should be added.

Rectum and recto-sigmoid junction. Rectal sigmoid nodules are visualised as an irregular hypoechoic mass penetrating into the intestinal wall distorting its normal structure At transvaginal sonography,

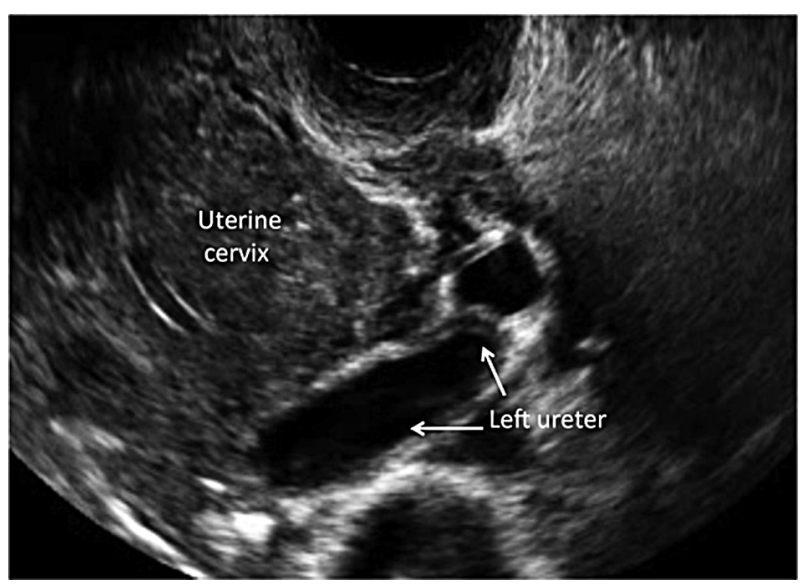

Fig. 8. Ultrasound image of a dilated ureter seen by transvaginal ultrasound in a transverse section of the pelvis, as tubular anechoic structure (arrows) in the parametrial tissue laterally to the uterine cervix. 


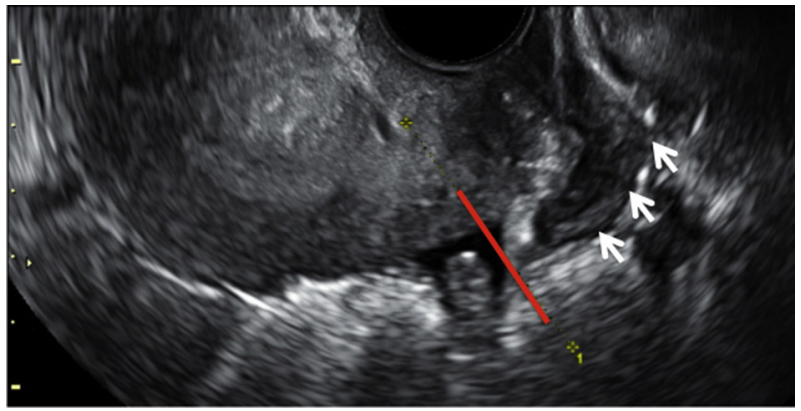

Fig. 9. Ultrasound appearance of a nodule of deep infiltrating endometriosis in the lower rectum (arrows). Nodules (arrows) located below the level of a virtual line (red line) passing through the insertion of the uterosacral ligaments on the cervix are considered to be located in the lower rectum, which are more difficult to be surgically resected compared with upper rectum or recto-sigmoid junction lesions.

the normal rectal wall layers are seen: the rectal serosa and smooth muscle layer appear as a thin, hypoechogenic line covered by the rectal submucosa and mucosa, which is visualised as a hyperechogenic rim covering the rectal smooth muscle layer [55]. With the posterior uterine wall, intestinal nodules located below the level of the insertion of the uterosacral ligaments (USLs) on the cervix are considered low rectal lesions (Fig. 9), whereas the ones above this level are considered upper rectal or the recto-sigmoid junction lesions (Fig. 10) This virtual line should delimit the plane under the peritoneum of the POD and correspond laterally to the parametria and medially to the recto-vaginal septum. Bazot et al. [47] described a similar plane as the horizontal plane passing through the lower border of the posterior lip of the cervix, which is lower and does not consider the posterior vaginal fornix as delimitation of the recto-vaginal septum. The diameters (e.g. longitudinal, transversal and antero-posterior) of each lesion should be taken; however, often, the irregular border does not permit accurate measurements to be taken. The distance from the anus can be taken by transrectal sonography, evaluating the distance from the anal opening on the outside of the body to the tip of the probe, positioning the tip of the probe on the endometriotic lesion.

Pouch of Douglas obliteration. Obliteratoin of the Pouch of Douglas s assessed using the sliding sign by gently exerting pressure on the cervix with the transvaginal probe to evaluate whether the anterior rectum glides freely across the posterior aspect of the cervix, or by placing the left hand over the

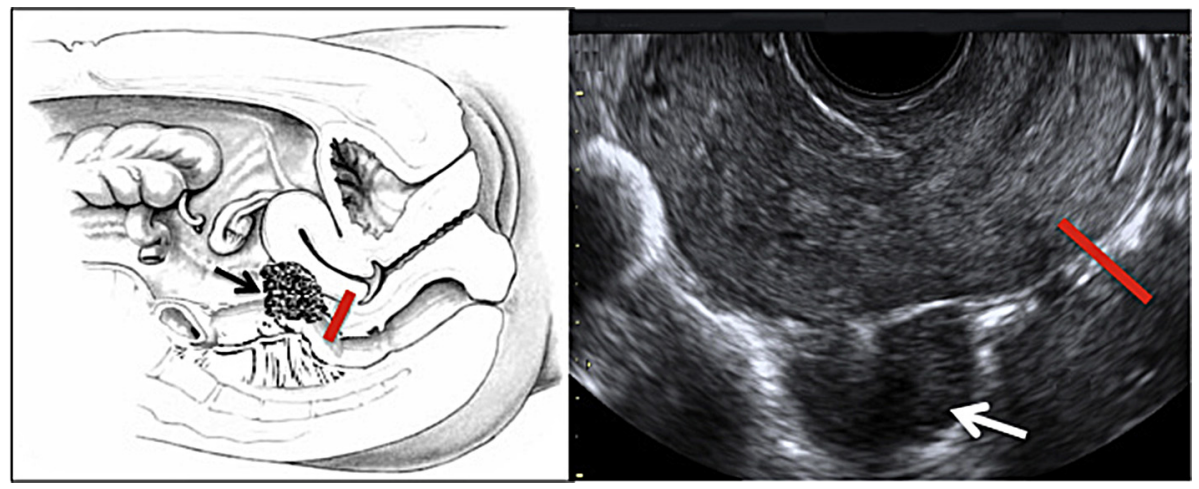

Fig. 10. Schematic and ultrasound image of a nodule of deep infiltrating endometriosis in the upper rectum (arrow). Nodules located above the level of a virtual line (red line) passing through the insertion of the uterosacral ligaments on the cervix are considered to be located in the upper rectum or recto-sigmoid junction. 
woman's lower anterior abdominal wall to ballot the uterus between the palpating hand and transvaginal probe to determine whether the recto sigmoid glides freely over the posterior aspect of the upper uterus or fundus [38,39].

Recent studies have shown that transvaginal sonography, when carried out by experienced sonographers, may indeed be a highly valuable test for the detection of DIE [47-50,56,57].

The reported accuracy of the ultrasound diagnosis of DIE varies between studies, which may reflect the variations in the examination technique, quality of ultrasound equipment, and experience of the operators. The prevalence of disease is also variable in different studies, which may bias the findings.

Although reported sensitivity and specificity of transvaginal sonography in the prediction of DIE is high [47-58], evaluation of DIE by transvaginal sonography is difficult and requires expertise. Therefore, some easily detectable utrasonographic signs have recently been proposed to predict the risk of the presence of DIE. Real-time dynamic transvaginal sonography evaluation of the posterior compartment using the 'sliding sign' seems to establish whether the pouch of Douglas is obliterated, and may also be useful in the identification of women who may be at a higher risk for bowel endometriosis $[38,39]$.

Transvaginal sonography has low accuracy in diagnosing the infiltration of the mucosal layer [4]. Also, transrectal ultrasound, which is a valuable tool for detecting rectal endometriosis as endometriotic infiltration of the muscularis layer, is less accurate in assessing submucosal, mucosal layer involvement, or both $[49,59]$. Therefore, transvaginal and transrectal sonography does not help surgeons in deciding whether or not to perform segmental or discoid resection of the lesion. More likely, this decision depends on the patient's symptoms and is also related to the diameters of infiltrating tissue and the presence of lumen stenosis. It has been reported that adding water-contrast in the rectum during transvaginal ultrasonography improves the diagnosis of rectal infiltration in women with rectovaginal endometriosis [60]. Saline solution is injected into the rectal lumen under ultrasonographic control through a catheter (Fig. 11). Presence of rectovaginal nodules, presence and degree of rectal infiltration, and the largest diameter of the bowel nodules can be evaluated. The procedure determines the presence of rectovaginal nodules infiltrating the rectal muscularis propria more accurately than transvaginal sonography [60]. It can be used when transvaginal sonography cannot exclude the presence of rectal infiltration. In cases of suspected bowel stenosis based on symptoms and on transvaginal sonography findings, a barium enema could help decide whether segmental resection is necessary.

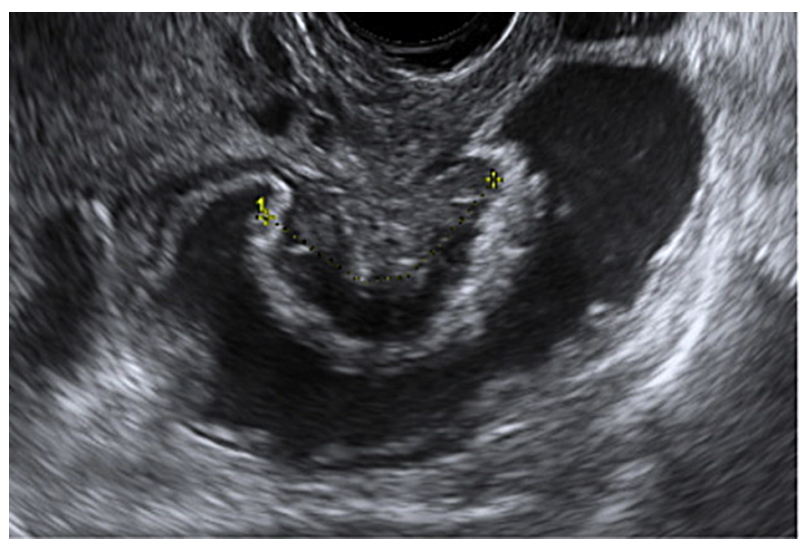

Fig. 11. Water-contrast in the rectum during transvaginal sonography is performed by injecting saline solution into the rectal lumen during transvaginal ultrasound examination. Note the presence of the deep infiltrating endometriosis nodule bulging into the bowel lumen. The lesion clearly reduces the rectal lumen. It infiltrates only the muscle layer of the bowel. The lesion is covered by the hyperechogenic submucosa and hypoechogenic mucosa. 


\section{Magnetic resonance imaging of deep infiltrating endometriosis}

Deep infiltrating endometriosis of the anterior compartment (bladder)

Magnetic resonance imaging shows localised thickening of the bladder wall with occasional protrusion inside the bladder lumen. In some cases, an extended plaque of fibrous tissue may invade the vesico-uterine pouch, completely obliterating it. In these cases, the tissue can be in contact with an adenomyotic nodule of the anterior wall of the uterus. T2-weighted images on the sagittal plane show hypointense tissue, irregular borders of the bladder, and sometimes the presence of tiny hyperintense spots inside the tissue corresponding to cystic glandular dilatation of the endometrial glands. On T1weighted images, hyperintense foci may be visualised; this finding is highly specific for the presence of endometriosis (Fig. 12).

In a study of 195 women with clinical suspicion of endometriosis, Bazot et al. [61] reported MRI sensitivity of $88 \%$, specificity of $99 \%$ (177 out of 179 ), and diagnostic accuracy of $98 \%$ in the diagnosis of bladder endometriosis.

An important question concerns possible encasement of the distal ureter requiring ureteral reimplantation during surgery. Direct invasion of the ureter causes luminal narrowing and may cause dilatation. Magnetic resonance urography is non-specific. Infiltration of the ureter should be suspected when the interface of fat between the nodule and the ureter is no longer visible on T2-weighted sequences. Hydronephrosis is easy to detect using magnetic resonance urography obtained with either 2D T2-weighted sequences or delayed contrast-enhanced three-dimensional sequences with higher spatial resolution.

\section{Deep infiltrating endometriosis of the posterior-lateral compartment}

Magnetic resonance imaging MRI can evaluate various signs of involvement of endometriosis in this region: (1) presence of macroscopic endometriosis implants ( $>5 \mathrm{~mm}$ ); (2) indirect signs of adhesions, such as disappearance of the fat tissue plane separating the different structures; (3) direct signs of adhesions are well displayed on T2 -weighted imaging as hypointense bands, with variable thickness that result in stretching and distortion of the surrounding organs; (4) uterosacral ligament involvement is suspected in cases of increased and inhomogeneous thickness associated with abnormal arciform appearance; (5) presence of specific signs of posterior cul-de-sac obliteration: retroflexed uterus; tethered appearance of the rectum in direction of the uterus; strands between the uterus and

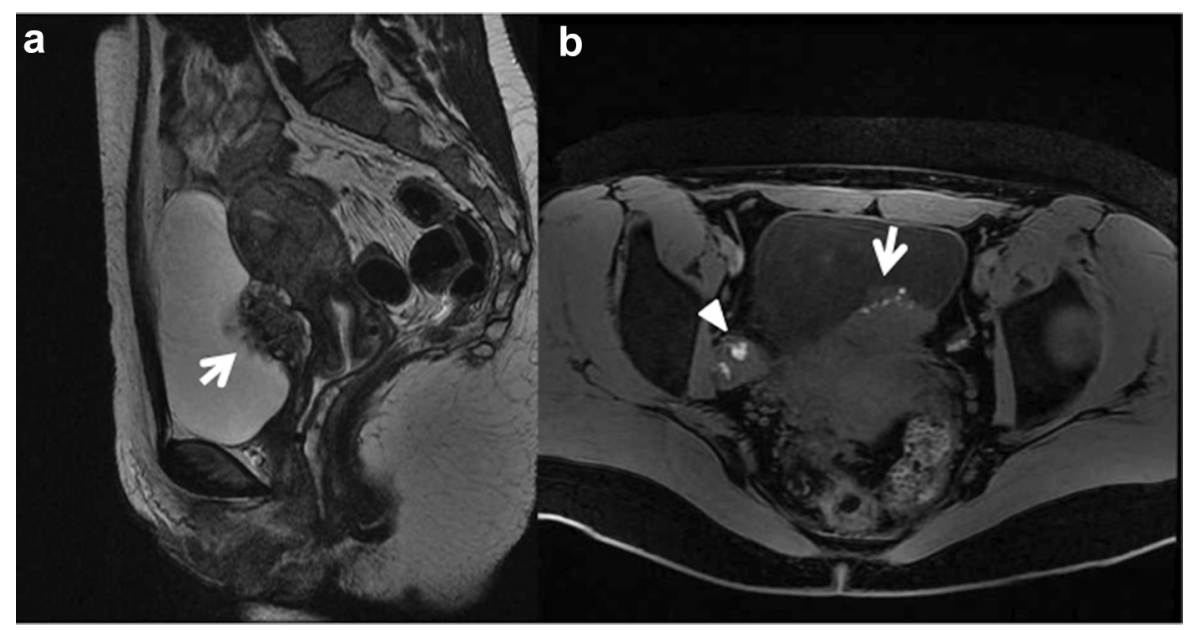

Fig. 12. Magnetic resonance image of infiltrating bladder endometriosis. Sagittal T2-weighted image showing obliteration of vesicouterine pouch and abnormal thickening of the posterior bladder wall (white arrow) (a). Axial T1 weighted image demonstrating the thickened abnormal bladder wall containing tiny hyperintense spots (white arrow). Endometriosis implants are visualised in right ovary (arrow head) (b). 
intestine; fibrotic plaque covering the serosal surface of the uterus and elevated posterior cervical fornix [40]; and (6) evaluation of signal intensity of endometriosis lesions is required as it varies according to the microscopic characteristics of the ectopic tissue. Signal intensity can present three main patterns: (1) hypointense signal on both T1- and T2-weighted sequences with hyperintense foci on T2weighted sequences, which may indicate fibrosis with glandular spots; (2) hypointense signal on T1weighted and T2-weighted images with hyperintense foci on T1-weighted image, which is caused by haemorrhagic foci within the fibrotic tissue; and (3) hypointense signal in both T1-weighted and T2weighted sequences if fibrotic reaction is abundant. This last feature may be missed on MRI or lead to a differential diagnosis.

The most commonly affected areas are in, decreasing order, the rectosigmoid colon, the appendix, the cecum, and the distal ileum. The lesions invade from serosa towards the bowel wall until muscolaris propria reacts with the hypertrophia and fibrosis. Bowel lesions are mainly fibromuscular, with occasional foci of T1- and T2-weighted hyperintensity. In these cases, the use of contrast media allows a better distinction between the lesion and the normal bowel wall. Diagnostic criteria of rectal invasion at MRI include colorectal wall thickening with anterior triangular attraction of the rectum toward the torus uteri or asymmetric wall thickening of the lower third of sigmoid colon (Fig. 13). Magnetic resonance imaging sensitivity and specificity of $84 \%$ and $99 \%$, respectively, have been reported in a study of 60 women with intestinal involvement [61]. These values are similar to those obtained using transvaginal ultrasound imaging in women with lesions located in the rectum. The main limitation of ultrasound imaging concerns lesions located above the rectosigmoid junction owing to the limited field-of-view of the transvaginal approach.

Other important data for using MRI relates to the multifocal behaviour of endometriosis. Unifocal isolated lesions of the intestine are reported in less than $21 \%$ of woman with intestinal lesions. The challenge of MRI, thanks to a large field of view, multiplanar capabilities, and outstanding contrast resolution, is to evaluate the entire pelvis and abdomen to detect associated lesions. Some conditions, however, can reduce the quality and sensitivity of magnetic resonance images. One of the most important conditions is bowel peristalsis, especially in women undergoing MRI to determine the presence of DIE of the intestine. Peristalsis blurs the bowel contours and adjacent organs, and may simulate bowel thickening or mask small lesions.

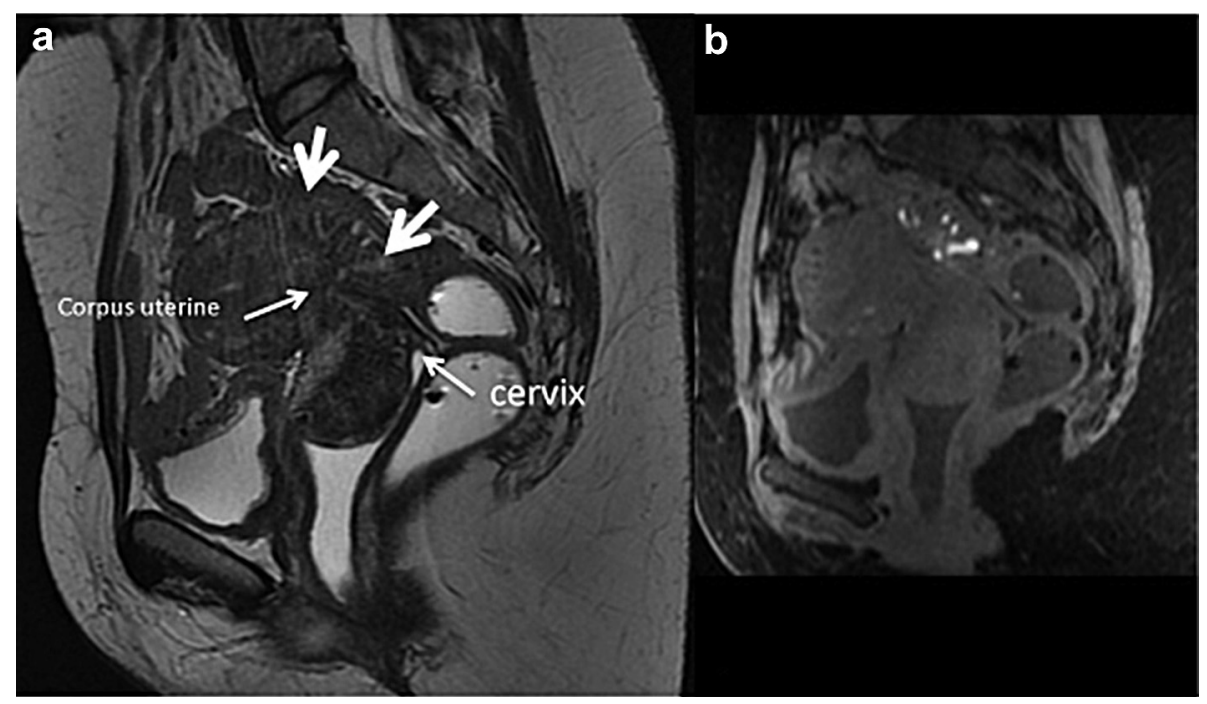

Fig. 13. Magnetic resonance imaging view of bowel endometriosis. (a) Sagittal T2-weighted image shows obliteration of posterior cul de sac and asymmetric wall thickening of the lower third of the sigmoid colon (white arrows). The endometriotic plaque infiltrates the sigmoid colon wall and also the uterine posterior wall, colon and uterus are therefore attached to each other. (b) Sagittal T1-weighted fat saturation image of the same case shows that the plaque contains hyperintense spots as for the presence of methaemoglobin suggestive for endometriosis. 
Kataoka et al. [40] reported 1.5 Tesla MRI sensitivity, specificity, accuracy, positive and negative predictive values of $68.4 \%, 76.0 \%, 71.9 \%, 76.6 \%, 68.5 \%$, respectively, in diagnosing posterior cul-de-sac obliteration.

Recently, some investigators have reported the role of 3.0 Tesla (3T) MRI in the evaluation of endometriosis. Pelvic 3T MRI guarantees a high spatial and contrast resolution, providing accurate information about endometriosis implants and a good pre-surgical mapping of lesions involving bowels, serosal surface of the bladder, and the utero-sacral ligaments [25,62]. Manganaro et al. [62] reported 3T MRI mean sensitivity, specificity, positive predictive value and negative predictive value of $93 \%, 75 \%, 93 \%, 75 \%$, respectively, in diagnosing DIE of the posterior compartment.

New modalities for the study of women with chronic pelvic pain are being developed using 3T MRI as described in a recent study dealing with the feasibility of diffusion tensor imaging and tractography in abnormalities of the sacral roots [63].

\section{Double-contrast barium enema}

Double-contrast barium enema (DBCE) has shown promising results when carried out by expert radiologists in the preoperative evaluation of women with clinically suspected intestinal (rectal, sigmoid, cecal) DIE [64]. It does, however, require a low-residue diet for 1 day before the examination, administration of drugs to empty the colon, and exposure to X-rays The presence of DIE is diagnosed on DCBE when the bowel lumen is narrowed at any level from the sigmoid to the anus (extrinsic mass effect) in association with crenulation of the mucosa, spiculation of contour, or both. Intestinal involvement in pelvic endometriosis that cause lumen stenosis can be evaluated by DCBE, which performs better than MRI. Its accuracy was reported at nearly $90 \%$ (sensitivity $88 \%$ and specificity $93 \%$ ), with a positive predictive value of $97 \%$ [64].

Savelli et al. [65] compared transvaginal sonography and DCBE in the diagnosis of bowel DIE in terms of presence or absence of the lesion. They reported a sensitivity for ultrasound of $91 \%$ and for DBCE $43 \%$; specificity was $100 \%$ and $100 \%$, positive predictive value was $100 \%$ and $100 \%$, negative predictive value was $29 \%$ and $6 \%$ and accuracy was $91 \%$ and $45 \%$, respectively. They found that transvaginal sonography had a much higher sensitivity than DCBE in detecting the presence of posterior DIE, and should thus be regarded as the imaging modality of choice when disease is clinically suspected. The method of choice for evaluating the degree of lumen stenosis present in bowel DIE is DCBE.

\section{Computed tomography}

Computed tomography plays a limited role in the evaluation of endometriosis.

Exposure to high levels of radiation and low specificity in the detection of this disease are the main limits. In selected cases, computed tomography may have a role to play in the evaluation of intestinal and ureteral endometriosis. In addition to this, computed tomography is the method of choice in the localisation of other sites of endometriosis, such as endometriosis of the thorax and of the diaphragm. With abdominal wall endometriosis, MRI is the main tool of imaging to characterise the lesions inside the umbilicus and the muscles. Also, transabdominal ultrasound with linear high-frequency probes is be able to see endometriotic lesions of the abdominal wall as hypoechoic tissue with irregular margins and few vessels.

Recently, some investigators have reported their experience using computed tomography colonography, with similar results to those of MRI. Computed tomography colonography detected luminal alteration of the rectosigmoid colon, achieving a sensitivity of $96.0 \%$ and a specificity of $48.0 \%$ $(P<0.001)$ in all women with rectosigmoid endometriosis, and a sensitivity of $84.0 \%$ and specificity of $80.0 \%(P=0.005)$ in women with DIE and cul-de-sac obliteration [66].

Other investigators [67] observed a high sensitivity and specificity in the assessment of the degree of bowel wall infiltration (sensitivity 100\%, specificity 97.6\%). In the same study, data relating to ureteral endometriosis were analysed (sensitivity $72.2 \%$, specificity $100 \%$ ). The investigators concluded that computed tomography is an accurate and reproducible technique but has to consider the patients' age, and therefore delivers a 'non-negligible radiation dose' [67]. 
Multidetector computed tomography enteroclysis urography allows radiologists to determine whether bowel endometriosis and ureteral compression are present without increasing the radiation dose imparted to the patient. Biscaldi et al. [68] reported a sensitivity of multidetector computed tomography enteroclysis urography in identifying ureteral compression of $97.1 \%$, a specificity of $98.8 \%$, and an accuracy of $99.0 \%$. Preliminary results [69] suggest that MRI urography is also accurate in differentiating between intrinsic and extrinsic forms of ureteral involvement; further studies are, however, required to define its role in directing better treatment.

Recently, some investigators [70] have reported their experience using [18F] fluorodeoxyglucose (FDG) positron emission tomography (PET) combined with CT (18FGD-PET-CT). In several benign diseases, measurement of hypermetabolism using FGD reflects the degree of inflammation and aggressiveness of the disease. In this preliminary study, the investigators did not observe hypermetabolic activity caused by endometriosis using 18FDG PET-CT. Because the target is non-specific, 18FDG for endometrial tissue [70] and the high radiation dose needed for PET-CT are actually limitations of this examination in young women of reproductive age.

\section{Adenomyosis}

Adenomyosis is a common gynaecologic disease characterised by the migration of endometrial glands and stroma from the basal layer of endometrium into the myometrium, and is associated with smooth muscle hyperplasia. Its frequency varies considerably in different studies, oscillating between 5 and $70 \%$ [71]. This great variability depends on the criteria used for diagnosis, the average age of the population studied, and the effort made searching for adenomyosis in the histological preparations [72].

Typical symptoms of adenomyosis are pain and bleeding, but many women remain asymptomatic. The diagnosis is usually based on histological findings in surgical specimens. Today, it is today widely accepted that the only two practical ways to reach a valid pre-surgical diagnosis are MRI and transvaginal sonography.

The cause of adenomyosis is unknown, but interesting theories have considered adenomyosis a pathology of the endo-myometrial junctional zone, and have attempted to explain the association of adenomyosis with subfertility and endometriosis $[73,74]$.

The endo-myometrial junctional zone

The junctional zone is a distinct, hormone-dependent uterine compartment at the endomyometrial interface, revealed more than 20 years ago by MRI [75]. It is also called archimyometrium or inner myometrium. On high-resolution transvaginal scan, the junctional zone is often visualised as a subendometrial hypoechoic 'halo'.

Despite the apparent lack of histological distinction between the junctional zone and the outer myometrium on light microscopy, these two zones are, in reality, structurally and biologically different [76]. In recent years, this endometrio-myometrial junctional zone has emerged as a specialised zone, which critically governs many reproductive functions [77,78].

Growing evidence suggests that disruption of the normal junctional zone architecture associated with thickening or hyperplasia of the junctional zone (which seems to precede adenomyosis) and adenomyosis, inevitably alters the coordinated peristaltic activity of the inner myometrium $[79,80]$. Dysfunctional- and hyper-peristalsis of the junctional zone may affect sperm transport and implantation, contributing to infertility $[81,82]$. These peristaltic disorders of the junctional zone have also been linked to dysmenorrhoea and menorrhagia, and may play a role in the pathogenesis of endometriosis by facilitating retrograde menstruation and implantation of viable endometrial cells into the abdominal cavity [73,81]. Pelvic endometriosis, especially in severe stages, is also strongly associated with junctional zone thickening $[73,74,83,84]$. Observational studies suggest that perturbations in junctional zone structure or functions before conception predispose towards a spectrum of fertility and obstetrical complications [85]. 
Ultrasound features of adenomyosis and junctional zone

The two-dimensional sonographic findings of adenomyosis that have been described are generally alterations of the outer myometrium. The two-dimensional transvaginal sonographic evaluation of the junctional zone seems also to be, with high-frequency probes (5-10 MHz), difficult and imprecise, and sonographic differentiation of inner and outer myometrium is not always optimal. Recently, it has been observed that, on the coronal section of the uterus obtained with three-dimensional transvaginal sonography, it is possible to visualise the junctional zone more clearly with some post-processing arrangements $[84,86]$.

On the basis of two-dimensional transvaginal sonography, features considered to be associated with adenomyosis are as follows [87]: globally enlarged uterus: the fundus of the uterus appears enlarged; round cystic area within the myometrium (Fig. 14); asymmetrically enlarged uterus (e.g. anterior wall thicker than posterior wall, or vice versa) unrelated to leiomyoma (Fig. 15); inhomogeneneous, irregular myometrial echotexture in an indistinctly defined myometrial area with decreased or increased echogenicity; myometrial hypoechoic linear striations seen as a radiating pattern of thin acoustic shadows not arising from echogenic foci or leiomyoma (Fig. 16); an indistinct, fuzzy endometrialmyometrial border (ill-defined endometrial stripe); and the presence of diffuse vascularity, seen as diffusely spread of small vessels, which do not follow the normal course of the arcuate and radial arteries inside the myometrium (Figs. 15 and 16).

Power Doppler can be used to distinguish myometrial cysts from blood vessels, and to discriminate between leiomyomas and focal adenomyosis. Uterine leiomyomas manifest a circular flow along the myoma capsule, whereas localised adenomyosis and adenomyomas are characterised by diffusely spread vessels inside the lesions.

Three-dimensional transvaginal sonographic signs of adenomyosis are based on the evaluation of the junctional zone on the acquired volume of the uterus to obtain the coronal view. On the coronal view, the junctional zone appears as a hypoechoic zone around the endometrium. Volume contrast imaging (VCI) with 2-4 mm slices is used to see clearly in all planes of the multiplanar view, and also in the longitudinal and transverse uterine section where the anterior and posterior junctional zone can be evaluated (Fig. 17).

Disruption and infiltration of the hypoechoic junctional zone by means of the hyperechoic endometrial tissues can be evaluated (Fig. 18). To avoid only subjective morphological evaluation of the junctional zone as irregularity and infiltration, the objective parameters as measurement of the thickness of the junctional zone that radiologists generally use on MRI has also been proposed for three-dimensional junctional zone assessment [88].

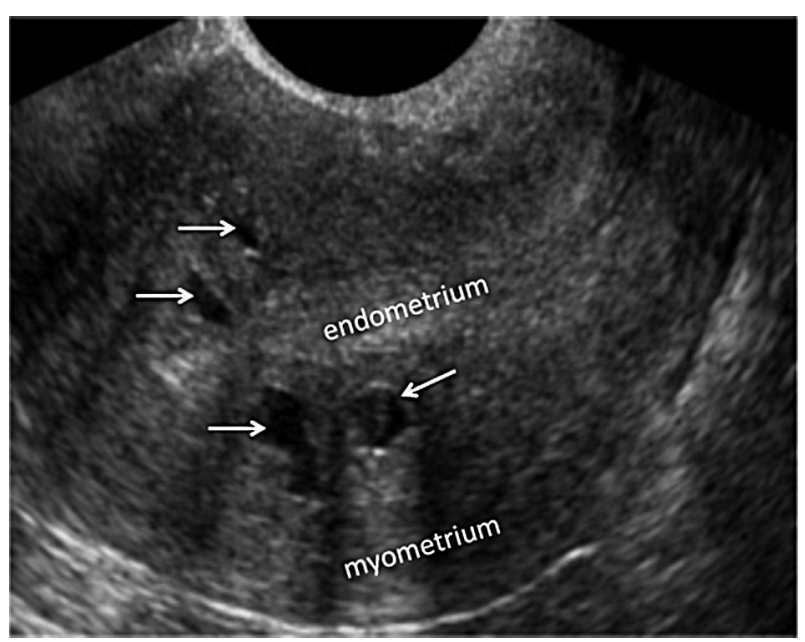

Fig. 14. Ultrasound image of a uterus with adenomyosis. Note the round cystic anechoic areas (arrows) in the myometrium below the endometrium in the junctional zone. 


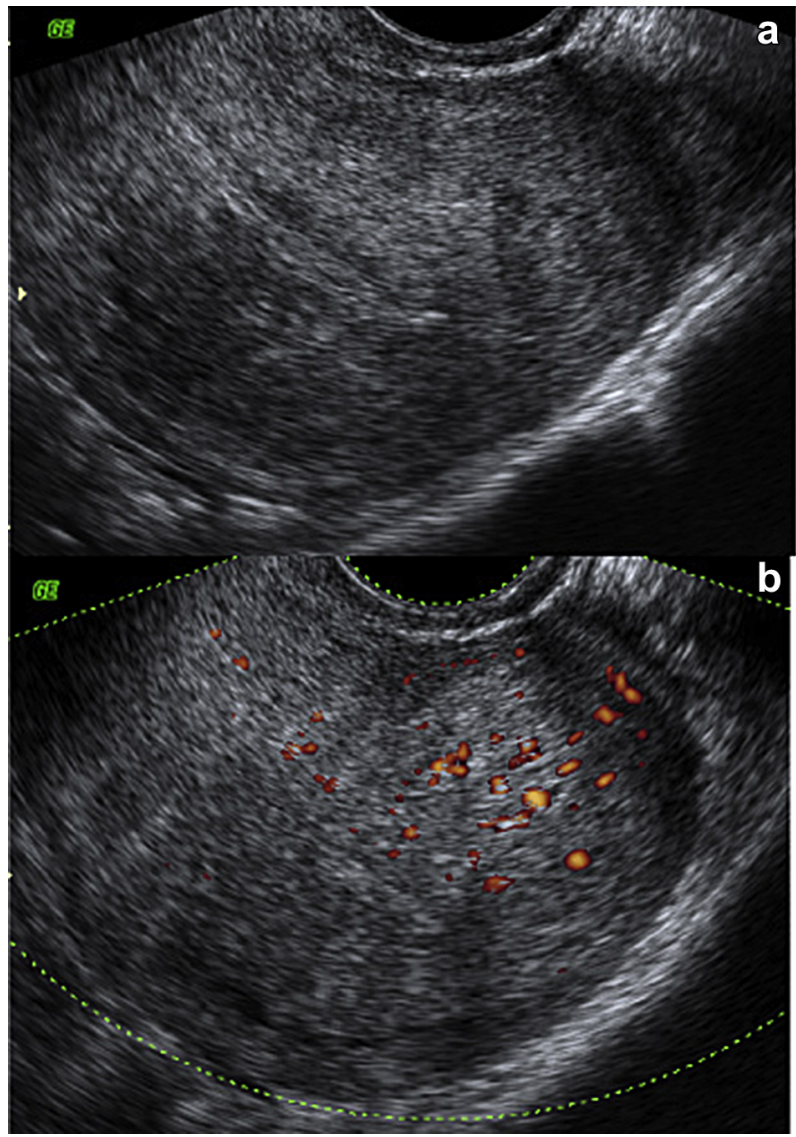

Fig. 15. Ultrasound images of a retroverted uterus with adenomyosis Gray scale image showing asymmetrically thickened posterior uterine wall with abnormal echogenicity (a). Power Doppler image showing diffusely spread small vessels (b).

The junctional zone can be measured on three-dimensional multiplanar view with volume contrast imaging modality; the greatest (maximal junctional zone thickness $=\mathrm{JZ}$ max), and lowest (minimal thickness of the junctional zone $=\mathrm{JZ}$ min) diameter of the junctional zone can be taken on coronal section or longitudinal section at any level of the uterus [88]. The advantage of measuring the junctional zone thickness is to have objective parameters for comparisons. Alterations of the junctional zone are defined as distortion and infiltration of the hypoechoic inner myometrium by hyperechoic endometrial tissue or as an ill-defined junctional zone.

Several studies have shown that the sensitivity and specificity of two-dimensional transvaginal scan in diagnosing adenomyosis are comparable to those of MRI, histology, or both, ranging from 75\%-88\% and 67\%-93\%, respectively [89-91]. Although MRI analyses features are based on junctional zone measurements and evaluation, two-dimensional ultrasound findings generally describe alterations of the outer myometrium, such as heterogenicity, hypertrophy, or presence of cystic areas. Only recently Kepkep et al. [92] included poor definition of the junctional zone in the assessment of the accuracy of various two-dimensional transvaginal scan findings in the diagnosis of adenomyosis. They found that poor definition of the junctional zone had a high specificity (82\%) but a low sensitivity (46\%) in its diagnosis.

Three-dimensional reconstruction of uterine anatomy in the coronal plane provides a new view of the junctional zone [86]. Three-dimensional transvaginal scan features were compared with histology of the uterus after hysterectomies, and it was shown that junctional zone thickness 

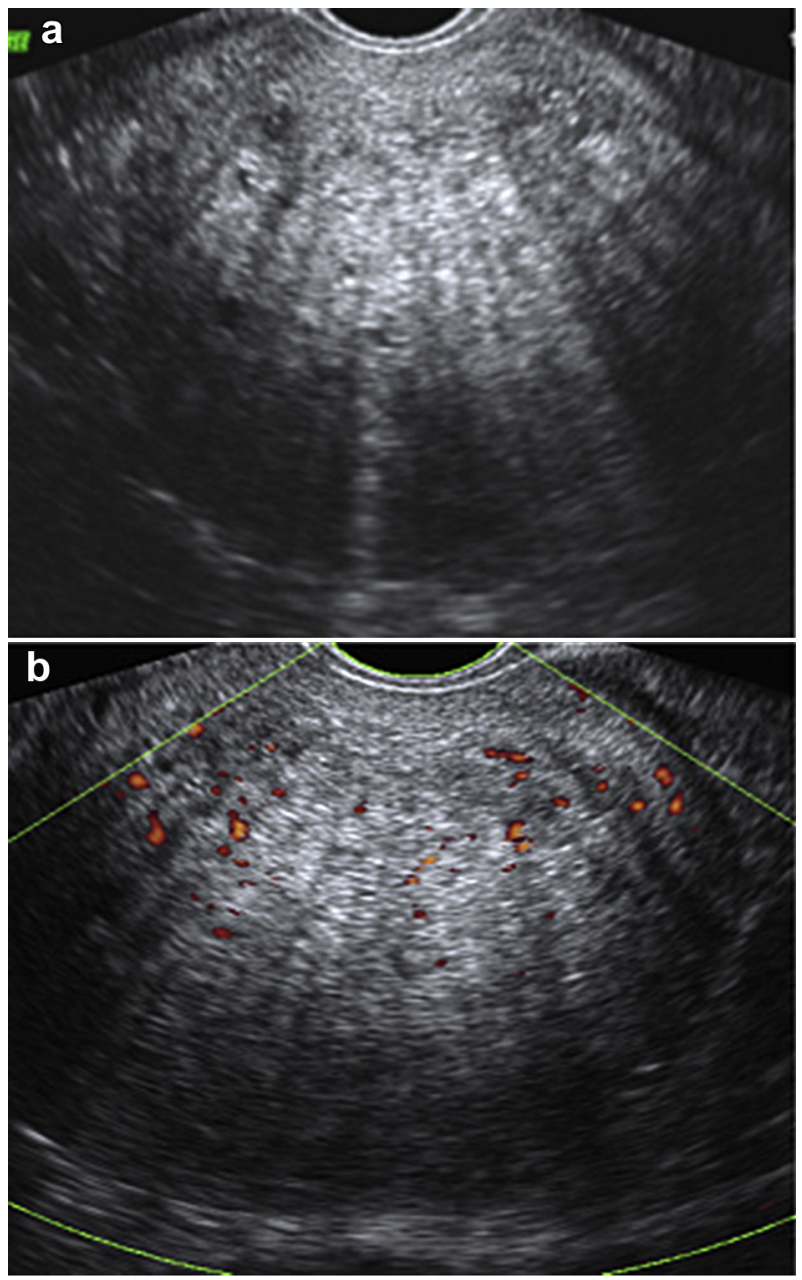

Fig. 16. Ultrasound images of a uterus in transverse section with adenomyosis. (a) Gray scale image showing inhomogeneous, irregular myometrial echotexture with hyperechoic irregular myometrial areas and a radiating pattern of linear striations; (b) colour Doppler image showing diffusely spread small vessels.

JZmax $\geq 8 \mathrm{~mm}$ and JZmax-JZmin $\geq 4 \mathrm{~mm}$ were significantly more associated with adenomyosis than other two-dimensional features [88,93]. Also, the subjective evaluation of infiltration and disruption by endometrial tissue in the junctional zone is an accurate tool for the diagnosis of adenomyosis $[86,88,93]$.

Thickening and disruption of the junctional zone appearance is strongly associated with uterine adenomyosis. Considering the hypothesis that adenomyosis is more likely to be caused by 'invasion' of endometrial tissue across the junctional zone and into the myometrium, three-dimensional transvaginal sonography evaluation of junctional zone could probably be able to detect initial adenomyosis. It has been observed that pelvic endometriosis, especially in severe stages, is also strongly associated with junctional zone thickening and adenomyosis $[83,84,88,94]$. The junctional zone, if altered, is correlated to adenomyosis, and seems to be involved in the process that determines pelvic endometriosis $[73,76,81,83,84]$.

Therefore, the evaluation of junctional zone and its alterations by non-invasive imaging seems important, especially in women with suspected pelvic endometriosis and adenomyosis. Three- 


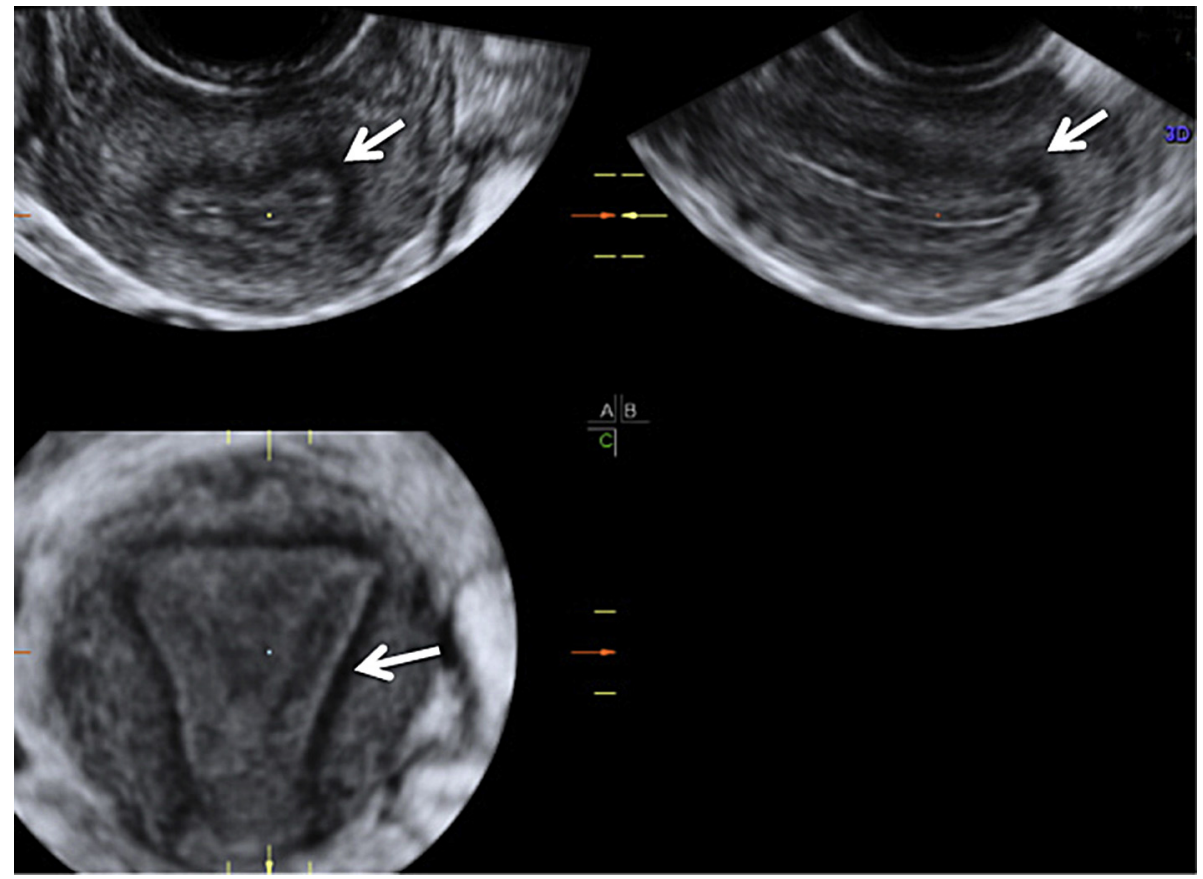

Fig. 17. Ultrasound image of the uterus obtained using three-dimensional ultrasound and volume contrast imaging with 4-mm slices. A multiplanar view is shown: transverse and coronal sections of the uterus are shown on the left side of the image, a longitudinal section is shown on the right side of the image. The junctional zone appears as a hypoechoic zone surrounding the endometrium (arrows).

dimensional transvaginal sonography seems to be more accurate than conventional two-dimensional sonography to detect adenomyosis, and could be proposed to evaluate early stages of the disease and considered in the treatment and counselling women with DIE.

Magnetic resonance imaging of adenomyosis and junctional zone

The MRI signs of adenomyosis are essentially linked to a thickening of a junctional zone. Typical adenomyosis appears as an ill-demarcated low-signal-intensity area on T2-weighted images. Furthermore on T2-weighted MRI, small high-signal-intensity areas refer to ectopic endometrium. Small cysts may also appear as high-signal-intensity spots on T2-weighted images (Fig. 19). Some of these areas may show hyperintense signals for the presence of methaemoglobin, on T1-weighted acquisitions fat saturation, which is a highly specific sign.

Subjective impressions, such as endometrial invasion and disruption of junctional zone irregularities seen at MRI imaging, are commonly used for diagnosis of adenomyosis [95], but objective criteria are preferable. Three objective parameters have been identified for MRI diagnosis of adenomyosis: the thickening of the junctional zone to at least $8-12 \mathrm{~mm}$ [89-91,95], the ratio junctional zone max-total myometrium over $40 \%$ [84,90], and the difference between the maximum and the minimum thickness of the junctional zone (JZmax-JZmin = JZ dif) more than $5 \mathrm{~mm}$ [91]. Although the first two criteria have been criticised because of changing hormonal status and menstrual cycle of junctional zone thickness [91], the third one seems to be more independent from hormonal status being a difference of two measurements both taken in the same hormonal phase.

Magnetic resonance imaging is also an accurate, non-invasive modality for diagnosing adenomyosis with a high specificity (67\%-99\%) and accuracy (85\%-95\%) [89-91,95-98]. 

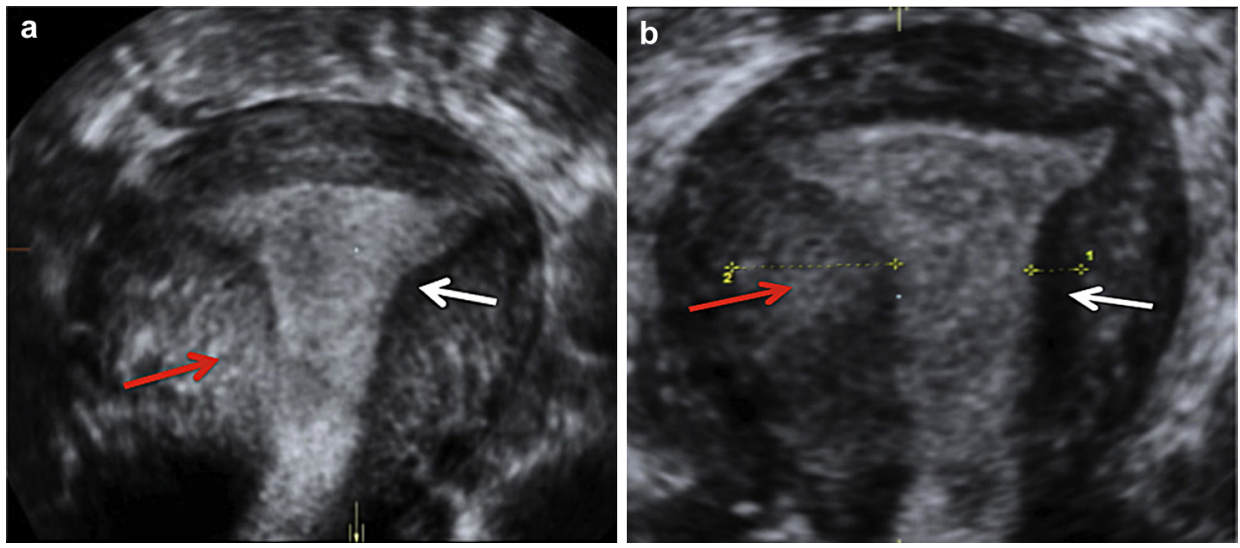

Fig. 18. Ultrasound image of the uterus with alteration of the junctional zone obtained in two different women using threedimensional ultrasound and volume contrast imaging. (a) A coronal view of the uterus is shown with the junctional zone appearing as a dark halo outside the endometrium on the right side of the image (white arrow) and with distortion and infiltration by hyperechoic endometrial tissue on the left side of the image (red arrow); (b) the normal (white arrow) and abnormal (red arrow). Junctional zones are measured on a coronal section through the uterus.

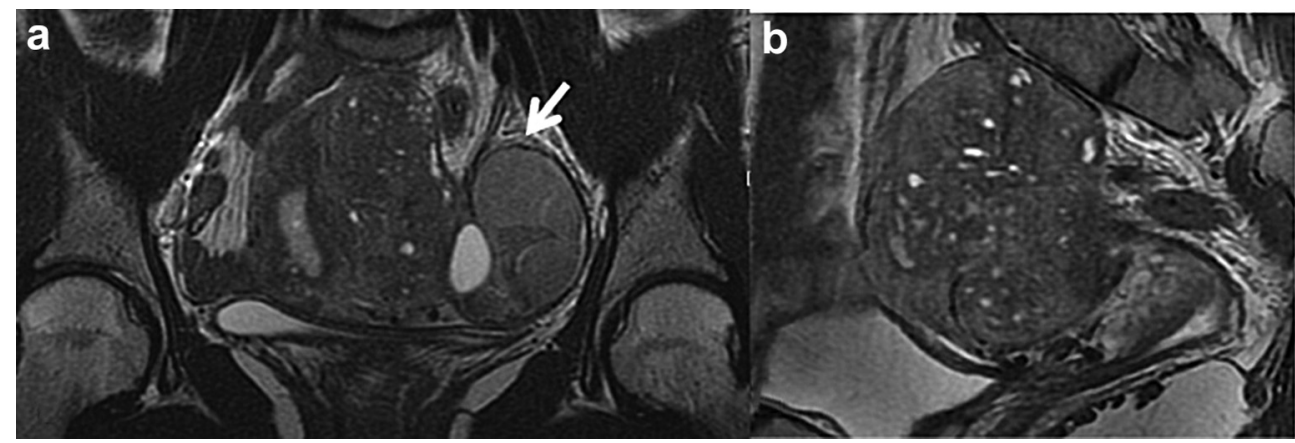

Fig. 19. Magnetic resonance view of adenomyosis: T2-weighted images on (a) coronal and (b) sagittal planes. Typical adenomyosis appears as an ill-demarcated, low-signal-intensity area on T2-weighted images. One can also see small high-signal-intensity areas corresponding to ectopic endometrium. Small cysts may also appear as high-signal-intensity spots on T2-weighted images. On the left side of the uterus, a dilated salpinx with endoluminal hypontense fluid suggestive of blood (white arrow).

\section{Conclusion}

In recent years, a number of studies have attempted to address the issue of diagnosing endometriosis, in particular by analysing the diagnostic performance of transvaginal sonography and magnetic resonance imaging, the two non-invasive methodologies most frequently used for the diagnosis of deep endometriosis.

The detection rate of imaging procedures is linked to the experience of the operator. The first-line modality is transvaginal sonography, which is deemed to be accurate in detecting both ovarian and extraovarian endometriosis. Magnetic resonance imaging has shown comparable results with those of transvaginal sonography in the assessment of deep endometriosis, but it is not as reliable as transvaginal sonography for the diagnosis of obliteration of the POD, which is crucial information for the surgeon $[49,56]$.

All MRI techniques may induce a potential discomfort for the patient, because of bowel preparation and the use of intravenous contrast medium. Also, the higher costs of carrying out MRI, and the need for expert radiologists, are a limitation of this diagnostic imaging for DIE diagnosis. The high accuracy in detecting and describing DIE lesions by transvaginal sonography is similar or better than those 
obtained by recent studies on magnetic resonance imaging $[56,99,100]$. For these reasons, it seems that transvaginal sonography is the best diagnostic tool in mapping DIE [36,48-50,57,58].

The multifocality of DIE lesions justifies a multidisciplinary diagnostic and treatment management. The possibility of intestinal lesions associated with gynecologic DIE requires close collaboration between gynaecologists, colorectal surgeons, and also urologists in the event of ureter involvement. In most cases, a reliable diagnosis can be made at gynaecological ultrasound evaluation by an experienced ultrasound examiner. A collaboration with radiologists is needed in unclear cases to establish a precise preoperative mapping of the DIE lesions.

The main issue is to identify which radiological examination is best associated with transvaginal sonography to diagnose DIE lesions. Although MRI is an efficient means of examination, allowing a complete pelvic work up to be established [101], bowel movements, notably in the sigmoid and ileocecal junction areas, may generate artifacts [48] that will hamper diagnosis, with the risk of overlooking and under-estimating the extent of intestinal DIE lesions.

So the basic question in daily practice is whether it is necessary to carry out MRI or other imaging like DBCE or computed tomography urography systematically in all women presenting a clinical suspicion of DIE. If intestinal infiltration is found at transvaginal sonography, we feel that MRI or DBCE is not always needed, given that it will provide no further information when lesions are small. If there is a strong clinical suspicion of intestinal involvement, however, but transvaginal sonography shows no intestinal infiltration, MRI could be useful to exclude lesions of the upper intestinal tract $[48,49,51]$.

Today, transvaginal sonography must be the first-line imaging process for women with suspected pelvic endometriosis.

\section{Practice points}

- Adhesions must be suspected if the ovaries and the uterus adhere to each other or to adjacent structures. This can be ascertained by palpating the ovaries and the uterus with the transvaginal probe, by palpating the abdoman with the hands, or both.

- Endometriosis may change the normal course of the Fallopian tube. Adhesions or endometriotic foci may occlude the Fallopian tube; consequently, a sactosalpinx may be seen on ultrasound or at magnetic resonance imaging, near an endometrioma or associated with deep lesions.

- All potential locations of DIE in the anterior (bladder) or posterior compartment (vagina, rectovaginal septum, torus uterinus and uterosacral ligaments, parametria, ureters, rectum, and recto-sigmoid junction) can be evaluated by transvaginal ultrasound and MRI with similar diagnostic accuracy; however, both imaging techniques need expert operators.

- Two-dimensional sonographic features of adenomyosis are changes in the myometrium, such as heterogeneity, hypoechoic linear striations, and cystic areas.

\section{Research agenda}

- To develop an ultrasound classification of pelvic endometriosis (e.g. terms, definition of features and sonographic anatomical markers) to be used by all staff involved in the diagnosis and treatment of women with endometriosis.

- To establish and validate simple and reproducible ultrasound signs of DIE and pelvic adhesions.

- To develop new MRI modalities for the detection of endometriotic lesions on nerve tracts and the peritoneal surface.

- To create a scoring system based on ultrasound or magnetic resonance findings to predict surgical difficulties in women with endometriosis and to validate it in centres with substantial experience in operating on women with endometriosis.

- To describe the ultrasound appearance of the junctional zone and adenomyosis in women with endometriosis and to explore whether its appearance is associated with infertility. 


\section{References}

[1] Giudice LC. Clinical practice. Endometriosis. N Engl J Med 2010;362:2389-98.

[2] Nnoaham KE, Hummelshoj L, Webster P, et al. Impact of endometriosis on quality of life and work productivity: a multicenter study across ten countries. Fertil Steril 2011;96:366-73.

[3] Koninckx PR, Meuleman C, Demeyere S, et al. Suggestive evidence that pelvic endometriosis is a progressive disease, whereas deeply infiltrating endometriosis is associated with pelvic pain. Fertil Steril 1991;55:759-65.

*[4] Koninckx PR, Ussia A, Adamyan L, et al. Deep endometriosis: definition, diagnosis, and treatment. Fertil Steril 2012;98: 564-71.

[5] Society AF: Revised American fertility society classification of endometriosis. Fertil Steril 1985;43:351-2.

[6] Hudelist G, Fritzer N, Thomas A, et al. Diagnostic delay for endometriosis in Austria and Germany: causes and possible consequences. J Hum Reprod 2012;27:3412-6.

[7] Kralik R, Trnovsky P, Kopàçovà M. Transabdominal ultrasonography of the small bowel. Gastroenterol Res Pract 2013; 2013:1-11.

[8] Van Gorp T, Amant F, Neven P, et al. Endometriosis and the development of malignant tumours of the pelvis. A review of literature. Best Pract Res Clin Obstet Gynaecol 2004;18:349-711.

[9] Kawaguchi R, Tsuji Y, Haruta S, et al. Clinicopathologic features of ovarian cancer in patients with ovarian endometrioma. J Obstet Gynaecol Res 2008;34:872-7.

[10] Testa AC, Timmerman D, Van Holsbeke C, et al. Ovarian cancer arising in endometrioid cysts: ultrasound findings. Ultrasound Obstet Gynecol 2011;38:99-106.

[11] Valentin L. Pattern recognition of pelvic masses by gray-scale ultrasound imaging: the contribution of Doppler ultrasound. Ultrasound Obstet Gynecol 1999;14:338.

[12] Timmerman D, Testa AC, Bourne T, et al. Logistic regression model to distinguish between the benign and malignant adnexal mass before surgery: a multicenter study by the International Ovarian Tumor Analysis Group. J Clin Oncol 2005;23:8794-801.

[13] Alabiso G, Alio L, Arena S, et al. Ovarian endometrioma: what the patient needs. J Minim Invasive Gynecol; 2014. http://dx.doi.org/10.1016/j.jmig.2014.01.011 [Epub ahead of print].

[14] Guerriero S, Mais V, Ajossa S, et al. Transvaginal ultrasonography combined with CA-125 plasma levels in the diagnosis of endometrioma. Fertil Steril 1996;65:293-9.

[15] Patel M, Feldstein V, Chen D, et al. Endometriomas: diagnostic performance of US. Radiology 1999;210:739-45.

[16] Valentin L. Use of morphology to characterize and manage common adnexal masses. Best Pract Res Clin Obstet Gynaecol 2004;18:71-89.

[17] Guerriero S, Ajossa S, Mais V, et al. The diagnosis of endometriomas using colour Doppler energy imaging. Hum Reprod 1998;6:1691-5.

[18] Dogan M, Ugur M, Soysalb S, et al. Transvaginal sonographic diagnosis of ovarian endometrioma. Int J Gyn Obstet 1996;52:145-9.

*[19] Van Holsbeke C, Van Calster B, Guerriero S, et al. Endometriomas: their ultrasound characteristics. Ultrasound Obstet Gynecol 2010;35:730-40.

[20] Lalwani N, Shanbhogue AK, Vikram R, et al. Current update on borderline ovarian neoplasms. AJR Am J Roentgenol 2010;194:330-6.

[21] Fruscella E, Testa AC, Ferrandina G, et al. Sonographic features of decidualized ovarian endometriosis suspicious for Malignancy. Ultrasound Obstet Gynecol 2004;24:578-80.

[22] Mascilini F, Moruzzi C, Giansiracusa C, et al. Imaging of gynecological disease: clinical and ultrasound characteristics of decidualized endometriomas surgically removed during pregnancy. Ultrasound Obstet Gynecol; 2014. http://dx.doi. $\operatorname{org} / 10.1002 /$ uog.13323 [Epub ahead of print].

[23] Timor-Tritsch IE, Lerner JP, Monteagudo A, et al. Transvaginal sonographic markers of tubal inflammatory disease. Ultrasound Obstet Gynecol 1998;12:56-66.

[24] Johnson N, van Voorst S, Sowter MC, et al. Surgical treatment for tubal disease in women due to undergo in vitro fertilisation. Cochrane Database Syst Rev; 2010 (1): CD002125.

[25] Hottat N, Larrousse C, Anaf V, et al. Endometriosis: contribution of 3.0-T pelvic MR imaging in preoperative assessment-initial results. Radiology 2009;253:126-34.

[26] Chamié LP, Blasbalg R, Pereira RM, et al. Findings of pelvic endometriosis at transvaginal US, MR imaging, and laparoscopy. Radiographics 2011;31:E77-100.

[27] Kim MY, Rha SE, Oh SN, et al. MR Imaging findings of hydrosalpinx: a comprehensive review. Radiographics 2009;29: 495-507.

[28] Chapron C, Pietin-Vialle C, Borghese B, et al. Associated ovarian endometrioma is a marker for greater severity of deeply infiltrating endometriosis. Fertil Steril 2009;92:453-7.

[29] Nezhat CR, Nezhat FR, Nezhat CH, et al. Treatment of ovarian endometriosis. In: Nezhat CR, Berger GS, Nezhat FR, editors. Endometriosis: advanced management and surgical technique. New York: Springer-Verlag; 1995. pp. 95-106.

[30] RCOG guideline: the investigation and management of endometriosis. Green-top guideline. 24, http://www.rcog.org. uk/files/rcog-corp/GTG2410022011.pdf; 2006 [last accessed 23.04.13].

[31] Redwine DB. Ovarian endometriosis: a marker for more extensive pelvic and intestinal disease. Fertil Steril 1999;72: $31-314$.

[32] Guerriero S, Ajossa S, Garau N, et al. Diagnosis of pelvic adhesions in patients with endometrioma: the role of transvaginal ultrasonography. Fertil Steril 2010;94:742-6.

[33] Okaro E, Condous G, Khalid A, et al. The use of ultrasound-based 'soft markers' for the prediction of pelvic pathology in women with chronic pelvic pain: can we reduce the need for laparoscopy? BJOG 2006;113:251-6.

*[34] Holland TK, Yazbek J, Cutner A, et al. The value of transvaginal ultrasound in assessing the severity of pelvic endometriosis. Ultrasound Obstet Gynecol 2010;36:241-8. 
[35] Exacoustos C, Zupi E, Carusotti C, et al. Staging of pelvic endometriosis: role of sonographic appearance in determining extension of disease and modulating surgical approach. J Am Assoc Gynecol Laparosc 2003;10:378-88.

*[36] Hudelist G, Oberwinkler KH, Singer CF, et al. Combination of transvaginal sonography and clinical examination for preoperative diagnosis of pelvic endometriosis. Hum Reprod 2009;24:1018-24.

[37] Khong SY, Bignardi T, Luscombe G, et al. Is pouch of Douglas obliteration a marker of bowel endometriosis? J Minim Invasive Gynecol 2011;18:333-7.

[38] Hudelist G, Fritzer N, Staettner S, et al. Uterine sliding sign: a simple sonographic predictor for presence of deep infiltrating endometriosis of the rectum. Ultrasound Obstet Gynecol 2013;41:692-5.

*[39] Reid S, Lu C, Casikar I, et al. Prediction of pouch of Douglas obliteration in women with suspected endometriosis using a new real-time dynamic transvaginal ultrasound technique: the sliding sign. Ultrasound Obstet Gynecol 2013;41: 685-91.

[40] Kataoka ML, Togashi K, Yamaoka T, et al. Posterior cul-de-sac obliteration associated with endometriosis: MR imaging evaluation. Radiology 2005;234:815-23.

[41] Fedele L, Bianchi S, Raffaelli R, et al. Pre-operative assessment of bladder endometriosis. Hum Reprod 1997;12:251922.

[42] Novellas S, Chassang M, Bouaziz J, et al. Anterior pelvic endometriosis: MRI features. Abdominal Imaging 2010;35: $742-9$.

[43] Fedele L, Bianchi S, Zanconato G, et al. Use of a levonorgestrel-releasing intrauterine device in the treatment of rectovaginal endometriosis. Fertil Steril 2001;75:485-8.

[44] Razzi S, Luisi S, Calonaci F, et al. Efficacy of vaginal danazol treatment in women with recurrent deeply infiltrating endometriosis. Fertil Steril 2007;88:789-94.

[45] Mabrouk M, Frascà C, Geraci E, et al. Combined oral contraceptive therapy in women with posterior deep infiltrating endometriosis. J Minim Invasive Gynecol 2011;18:470-4.

[46] Vercellini P, Somigliana E, Consonni D, et al. Surgical versus medical treatment for endometriosis-associated severe deep dyspareunia: I. Effect on pain during intercourse and patient satisfaction. Hum Reprod 2012;27:3450-9.

[47] Bazot M, Thomassin I, Hourani R, et al. Diagnostic accuracy of transvaginal sonography for deep pelvic endometriosis. Ultrasound Obstet Gynecol 2004;24:180-5.

[48] Abrao MS, Goncalves MO, Dias Jr JA, et al. Comparison between clinical examination, transvaginal sonography and magnetic resonance imaging for the diagnosis of deep endometriosis. Hum Reprod 2007;22:3092-7.

*[49] Piketty M, Chopin N, Dousset B, et al. Preoperative work-up for patients with deeply infiltrating endometriosis: transvaginal ultrasonography must definitely be the first-line imaging examination. Hum Reprod 2009;24:602-7.

[50] Hudelist G, Ballard K, English J, et al. Transvaginal sonography vs. clinical examination in the preoperative diagnosis of deep infiltrating endometriosis. Ultrasound Obstet Gynecol 2011;37:480-7.

[51] Chapron C, Fauconnier A, Vieira M, et al. Anatomical distribution of deeply infiltrating endometriosis: surgical implications and proposition for a classification. Hum Reprod 2003;18:157-61.

[52] Guerriero S, Ajossa S, Gerada M, et al. Diagnostic value of transvaginal 'tenderness-guided' ultrasonography for the prediction of location of deep endometriosis. Hum Reprod 2008;23:2452-7.

[53] Bignardi T, Condous G. Sonorectovaginography: a new sonographic technique for imaging of the posterior compartment of the pelvis. J Ultrasound Med 2008;27:1479-83.

[54] Pateman K, Mavrelos D, Hoo WL, et al. Visualization of ureters on standard gynecological transvaginal scan: a feasibility study. Ultrasound Obstet Gynecol 2013;41:696-701.

[55] Hudelist G, Tuttlies F, Rauter G, et al. Can transvaginal sonography predict infiltration depth in patients with deep infiltrating endometriosis of the rectum? Hum Reprod 2009;24:1012-7.

[56] Vimercati A, Achilarre MT, Scardapane A, et al. Accuracy of transvaginal sonography and contrast-enhanced magnetic resonance-colonography for the presurgical staging of deep infiltrating endometriosis. Ultrasound Obstet Gynecol 2012;40:592-603.

[57] Holland TK, Cutner A, Saridogan E, et al. Ultrasound mapping of pelvic endometriosis: does the location and number of lesions affect the diagnostic accuracy? a multicentre diagnostic accuracy study. BMC Womens Health 2013;13:4351.

[58] Fratelli N, Scioscia M, Bassi E, et al. Transvaginal sonography for preoperative assessment of deep endometriosis. J Clin Ultrasound 2013:41:69-75.

[59] Rossi L, Palazzo L, Yazbeck C, et al. Can rectal endoscopic sonography be used to predict infiltration depth in patients with deep infiltrating endometriosis of the rectum? Ultrasound Obstet Gynecol 2014;43:322-7.

[60] Valenzano Menada M, Remorgida V, Abbamonte LH, et al. Does transvaginal ultrasonography combined with watercontrast in the rectum aid in the diagnosis of rectovaginal endometriosis infiltrating the bowel? Hum Reprod 2008; 23:1069-75.

*[61] Bazot M, Darai E, Hourani R, et al. Deep pelvic endometriosis: MR imaging for diagnosis and prediction of extension of disease. Radiology 2004;232:379-89.

[62] Manganaro L, Vittori G, Vinci V, et al. Beyond laparoscopy: 3-T magnetic resonance imaging in the evaluation of posterior cul-de-sac obliteration. Magn Reson Imaging 2012;3:1432-8.

[63] Manganaro L, Porpora MG, Vinci V, et al. Diffusion tensor imaging and tractography to evaluate sacral nerve root abnormalities in endometriosis-related pain: a pilot study. Eur Radiol 2014;24:95-101.

[64] Faccioli N, Foti G, Manfredi R, et al. Evaluation of colonic involvement in endometriosis: double-contrast barium enema versus magnetic resonance imaging. Abdom Imaging 2010;35:414.

[65] Savelli L, Manuzzi L, Coe M, et al. Comparison of transvaginal sonography and double-contrast barium enema for diagnosing deep infiltrating endometriosis of the posterior compartment. Ultrasound Obstet Gynecol 2011;38:466-71.

[66] Jeong SY, Chung DJ, Myung Yeo D, et al. The usefulness of computed tomographic colonography for evaluation of deep infiltrating endometriosis: comparison with magnetic resonance imaging. J Comput Assist Tomogr 2013;37: 809-14. 
[67] Iosca S, Lumia D, Bracchi E, et al. Multislice computed tomography with colon water distension (MSCT-c) in the study of intestinal and ureteral endometriosis. Clin Imaging 2013;37:1061-8.

[68] Biscaldi E, Ferrero S, Remorgida V, et al. MDCT enteroclysis urography with split-bolus technique provides information on ureteral involvement in patients with suspected bowel endometriosis. AJR Am J Roentgenol 2011;196:635-40.

[69] Ghezzi F, Cromi A, Bergamini V, et al. Management of ureteral endometriosis: areas of controversy. Curr Opin Obstet Gynecol 2007;19:319-24.

[70] Fastrez M, Nogarede C, Tondeur M, et al. Evaluation of 18FDG PET-CT in the diagnosis of endometriosis: a prospective study. Reprod Sci 2011;18:540-4.

[71] Azziz R. Adenomyosis: current perspectives. Obstet Gynecol Clin North Am 1989;16:221-35.

[72] Bromley B, Shipp TD, Benacerraf B. Adenomyosis: sonographic findings and diagnostic accuracy. J Ultrasound Med 2000;19:529-34.

*[73] Leyendecker G, Wildt L, Mall G. The pathophysiology of endometriosis and adenomyosis: tissue injury and repair. Arch Gynecol Obstet 2009;280:529-38.

[74] Kunz G, Beil D, Huppert P, et al. Adenomyosis in endometriosis- prevalence and impact on fertility. Evidence from magnetic resonance imaging. Hum Reprod 2005;20:2309-16.

[75] Benagiano G, Brosens I. History of adenomyosis. Best Pract Res Clin Obstet Gynaecol 2006;20:449-63.

[76] Mehasseb MK, Bell SC, Pringle JH, et al. Uterine adenomyosis is associated with ultrastructural features of altered contractility in the inner myometrium. Fertil Steril 2010;93:2130-6.

[77] Brosens JJ, Barker FG, de Souza NM. Myometrial zonal differentiation and uterine junctional zone hyperplasia in the non-pregnant uterus. Hum Reprod Update 1998;4:496-502.

[78] Fusi L. The uterine junctional zone. Best Pract Res Clin Ob Gyn 2006;20:479-91.

[79] Kunz G, Beil D, Huppert P, et al. Structural abnormalities of uterine wall in women with endometriosis and infertility visualized by vaginal sonography and magnetic resonance imaging. Hum Reprod 2000;15:76-82.

[80] Lesny P, Killick SR. The junctional zone of the uterus and its contractions. BJOG 2004;111:1182-9.

[81] Leyendecker G, Kunz G, Herbertz M, et al. Uterine peristaltic activity and development of endometriosis. Ann NY Acad Sci 2004;1034:338-55.

[82] Maubon A, Faury A, Kapella M, et al. Uterine junctional zone at magnetic resonance imaging: a predictor of in vitro fertilization implantation failure. J Obstet Gynaecol Res 2010;36:611-8.

[83] Larsen SB, Lundorf E, Forman A, et al. Adenomyosis and junctional zone changes in patients with endometriosis. Eur J Obstet Gynecol Reprod Biol 2011;157:206-11.

[84] Exacoustos C, Luciano D, Corbett B, et al. The uterine junctional zone: a 3-dimensional ultrasound study of patients with endometriosis. Am J Obstet Gynecol 2013;209:248-55.

[85] Brosens I, Derwig I, Brosens J, et al. The enigmatic uterine junctional zone: the missing link between reproductive disorders and major obstetrical disorders? Hum Reprod 2010;3:569-74.

[86] Naftalin J, Jurkovic D. The endometrial-myometrial junction: a fresh look at a busy crossing. Ultrasound Obstet Gynecol 2009;34:1-11.

[87] Dueholm M. Transvaginal ultrasound for diagnosis of adenomyosis: a review. Best Pract Res Clin Obstet Gynaecol 2006;20:569-82.

*[88] Exacoustos C, Brienza L, Di Giovanni A, et al. Adenomyosis: three-dimensional sonographic findings of the junctional zone and correlation with histology. Ultrasound Obstet Gynecol 2011;37:471-9.

*[89] Dueholm M, Lundorf E. Transvaginal ultrasound or MRI for diagnosis of adenomyosis. Curr Opin Obset Gynecol 2007 19:505-12.

[90] Bazot M, Cortez A, Darai E, et al. Ultrasonography compared with magnetic resonance imaging for the diagnosis of adenomyosis: correlation with histopathology. Hum Reprod 2001;16:2427-33.

[91] Dueholm M, Lundorf E, Hansen ES, et al. Magnetic resonance imaging and transvaginal ultrasonography for diagnosis of adenomyosis. Fertil Steril 2001;76:588-94.

[92] Kepkep K, Tuncay YA, Goynumer G, et al. Transvaginal sonography in the diagnosis of adenomyosis: which findings are most accurate? Ultrasound Gynecol Obstet 2007;30:341-5.

[93] Luciano DE, Exacoustos C, Albrecht L, et al. Three-dimensional ultrasound in diagnosis of adenomyosis: histologic correlation with ultrasound targeted biopsies of the uterus. J Minim Invasive Gynecol 2013;20:803-10.

[94] Lazzeri L, Di Giovanni A, Exacoustos C, et al. Preoperative and postoperative clinical and transvaginal ultrasound findings of adenomyosis in patients with deep infiltrating endometriosis. Reprod Sci; 2014 [Epub ahead of print].

[95] Reinhold C, Tafazoli F, Wang L. Imaging features of adenomyosis. Hum Reprod Update 1998;4:337-49.

[96] Tamai K, Togashi K, Ito T, et al. MR imaging findings of adenomyosis: correlation with histopathologic features and diagnostic pitfalls. Radiographics 2005;25:21-40.

[97] Stamatopoulos CP, Mikos T, Grimbizis GF, et al. Value of magnetic resonance imaging in diagnosis of adenomyosis and myomas of the uterus. J Minim Invasive Gynecol 2012;19:620-6.

[98] Moghadam R, Lathi RB, Shahmohamady B, et al. Predictive value of magnetic resonance imaging in differentiating between leiomyoma and adenomyosis. JSLS 2006;10:216-9.

[99] Kruger K, Behrendt K, Niedobitek-Kreuter G, et al. Location-dependent value of pelvic MRI in the preoperative diagnosis of endometriosis. Eur J Obstet Gynecol Reprod Biol 2013;169:93-8.

*[100] Saba L, Sulcis R, Melis GB, et al. Diagnostic confidence analysis in the magnetic resonance imaging of ovarian and deep endometriosis: comparison with surgical results. Eur Radiol 2014;24:335-43.

[101] Kinkel K, Frei KA, Balleyguier C, et al. Diagnosis of endometriosis with imaging: a review. Eur Radiol 2006;16:285s298. 